\title{
Substituent Effect on the Photoreduction Kinetics of
}

\section{Benzophenone}

Attila Demeter, ${ }^{1} *$ Klaudia Horváth, ${ }^{l}$ Katalin Böör,${ }^{1}$ Laura Molnár,${ }^{2}$ Tibor Soós, ${ }^{2} *$ and György $\operatorname{Lendvay}^{1 *}$

Institute of Materials and Environmental Chemistry, ${ }^{1}$ Institute of Organic Chemistry, ${ }^{2}$ Research Centre for Natural Sciences, Hungarian Academy of Sciences, 1025 Budapest, Pusztaszeri u. 5967, Hungary

The kinetics of the photoreduction of four benzophenone derivatives by isopropyl alcohol was examined in acetonitrile, namely tetra-meta-trifluoromethyl-, di-para-trifluoromethyl-, di-paramethoxy benzophenone and for comparison the unsubstituted molecule itself. The basic spectroscopic (absorption and phosphorescence spectra) and photophysical (quantum yields, excited state energies) properties were established, and the key kinetic parameters were determined by the laser flash photolysis transient absorption technique. The rate coefficients of both the primary and secondary photoreduction reaction show remarkable dependence on ring substitution. This substantial effect is caused by the considerable change in the activation energy of the corresponding process. The experimental results as well as DFT quantum chemical calculations clearly indicate that these benzophenone derivatives all react as $n-\pi^{*}$ excited ketones, and the rate as well as the activation energy of the reduction steps change parallel with the reaction enthalpies, the determining factor being the stability of the forming aromatic ketyl 
radicals. The secondary photoreduction of benzophenones by the aliphatic ketyl radical formed in the primary step occurs via a hydrogen bonded complex. The binding energy of the hydrogen bonded complex between the aliphatic ketyl radical reactant and a solvent molecule is a critical parameter influencing the observable rate of the secondary photoreduction.

\section{INTRODUCTION}

The investigation of the photoreactions of benzophenone, especially the photoreduction by aliphatic alcohols is of historic importance. The earliest studies of the reaction served as a basis for the foundation of modern molecular photochemistry. In his pioneering work, ${ }^{1}$ Giacomo Ciamician explored the basic photochemical features of the benzophenone - ethanol system. He found that after a few days of solar irradiation an almost quantitative amount of benzopinacol was crystallized from the self-deoxygenated ethanolic solution of benzophenone. He also identified the oxidized side product, acetaldehyde.

Using 2-propanol as reducing agent, the dimethyl- and diaryl ketyl radicals (semipinacol and semibenzopinacol, respectively) are formed in equal amount, and they are consumed by self- and cross termination reactions. ${ }^{2,3}$ Moreover, it was recognized quite early ${ }^{4}$ that a radical transformation reaction may convert the primary intermediate, the aliphatic ketyl radical to the aromatic one, resulting in a benzophenone consumption quantum yield of almost two (and accordingly, in benzopinacol formation yield of nearly one) in ideal circumstances. This metathesis reaction makes the system an excellent candidate for photochemical preparation of benzopinacol and related compounds. ${ }^{5}$

Numerous properties of the primary photoreduction process, hydrogen abstraction by triplet $n-\pi^{*}$ state ketones are well known and have been discussed in detail..$^{6-12}$ The $n-\pi^{*}$ triplet state was found to behave as a 1,2-biradical in several types of chemical reaction. ${ }^{7,12}$ A notable number of 
studies $^{8}$ dealt with the nature of the primary photochemical process of $n-\pi^{*}$ excited aromatic carbonyls, mostly focusing on the dependence of the rate parameters on the chemical nature of the reaction partner. ${ }^{8,10,11}$ In reactions with hydrocarbons or alcohols the triplet excited ketones are considered $^{6,8}$ to behave like radicals, although the possibility of partial charge transfer character of the process has also been mentioned. ${ }^{8}$ The remarkable difference in the room temperature photoreduction rate was explained on the basis of the bond strength of the $\mathrm{C}-\mathrm{H}$ bond broken in the reaction. ${ }^{8}$ The possibility of hydrogen atom tunneling has also been suggested. ${ }^{9}$ Less attention has been paid to the dependence of the photoreduction rate on the chemical nature of the ketones themselves. It is commonly accepted that for hydrogen abstraction reactions, $\mathrm{n}-\pi^{*}$ excitation is much more favorable than $\pi-\pi *$ excitation. For ring-substituted acetophenone derivatives a large variance in photoreduction reactivity was observed. The tendencies were proposed to result from the changes of the reaction enthalpy, and partly from and the changing magnitude of mixing of the nearby $n-\pi^{*}$ and $\pi-\pi^{*}$ excited states. ${ }^{8,12}$ In addition, the lifetime of the triplet state as a measure of the photoreduction rate was determined in cyclohexane solvent at room temperature for various ring substituents of benzophenone and acetophenone. Good correlation was found between the reaction rate and the Hammett $\sigma$ parameter of the substituent. $^{8 b, 11}$

The character of the secondary photoreduction step (i.e. the radical conversion, reaction (5) in Scheme 1), although is very exciting from the theoretical point of view, is much less documented. The early experiments addressing the determination of the rate parameters were indirect and their results show high scatter (see Refs. 12, 13 and references therein). The first direct measurement of the rate parameter was made in our laboratory. ${ }^{13}$ Some controversy arose concerning the nature of the process. Naguib at al. ${ }^{14}$ suggested that the reaction takes place via 
concerted, spatially separated electron-proton transfer. Our earlier results ${ }^{13}$ indicate that, instead of such a special mechanism, the reaction should be considered to be a regular hydrogen atom metathesis process that takes place through a hydrogen bonded precursor complex. The primary aim of this study is to identify the major factors determining the rate of the secondary photoreduction steps in the most classical model system, the photoreduction of benzophenone by 2-propanol. We also intend to understand details of the mechanism of the reaction, such as the role the unavoidable hydrogen-bonded complex formation as well as the possibility of spatially separated electron-proton transfer. To this end, we studied a series of derivatives of the parent ketone, benzophenone in which ring substitution was applied to control the reactivity, in particular, the Arrhenius parameters of the $\mathrm{H}$ atom transfer rate coefficients. Both electron donating and electron withdrawing substituents, methoxy and trifluoromethyl, were applied in para position, having Hammett constants of -0.54 and 1.07 , respectively. Furthermore, to generate a more profound effect, we synthesized $3,3^{\prime}, 5,5^{\prime}-$ tetrakis(trifluoromethyl)benzophenone (CFBp, Scheme 2) for which the cumulative Hammett constant is $\sigma=1.72$. This derivative proved to be a versatile, easy to prepare aromatic ketone that has several promising features for use in further photochemical kinetic studies. (We note that trifluoromethyl-substituents have been recently demonstrated to be simple, cheap smallmolecular-weight phase labels that facilitate the separation of a reagent or catalyst from the reaction mixture. ${ }^{15}$ )

\section{The reaction mechanism}

The observations made in this study can be interpreted in terms of the simplified reaction mechanism presented in Scheme 1 for the benzophenone - 2-propanol system: ${ }^{13,16}$ 


\section{Scheme 1.}

$\mathrm{Ph}_{2} \mathrm{CO}+\mathrm{h} \mathbf{} \rightarrow{ }^{1} \mathrm{Ph}_{2} \mathrm{CO} \rightarrow{ }^{3} \mathrm{Ph}_{2} \mathrm{CO}$

${ }^{3} \mathrm{Ph}_{2} \mathrm{CO} \rightarrow \mathrm{Ph}_{2} \mathrm{CO}+$ had

${ }^{3} \mathrm{Ph}_{2} \mathrm{CO} \rightarrow \mathrm{Ph}_{2} \mathrm{CO}$

$2{ }^{3} \mathrm{Ph}_{2} \mathrm{CO} \rightarrow(2-\mathbf{\square}) \mathrm{Ph}_{2} \mathrm{CO}+\mathbf{\square}{ }^{3} \mathrm{Ph}_{2} \mathrm{CO}$

${ }^{3} \mathrm{Ph}_{2} \mathrm{CO}+\left(\mathrm{CH}_{3}\right)_{2} \mathrm{CHOH} \rightarrow \mathrm{Ph}_{2} \mathbf{D O H}+\left(\mathrm{CH}_{3}\right)_{2} \mathbf{D O H}$

$2\left(\mathrm{CH}_{3}\right)_{2} \mathrm{aOH} \rightarrow$ products

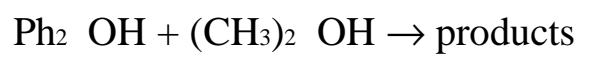

$\mathrm{Ph}_{2} \mathrm{CO}+\left(\mathrm{CH}_{3}\right)_{2} \mathbf{a O H} \rightarrow \mathrm{Ph}_{2} \mathbf{\square O H}+\left(\mathrm{CH}_{3}\right)_{2} \mathrm{CO}$

$2 \mathrm{Ph}_{2} \mathbf{D O H} \rightarrow \mathrm{Ph}_{2} \mathrm{C}(\mathrm{OH}) \mathrm{C}(\mathrm{OH}) \mathrm{Ph}_{2}$

After absorbing a photon, triplet benzophenone is formed in a very efficient intersystem crossing (ISC) process with a quantum yield of unity (with a characteristic time of 6-10 $\mathrm{ps}^{17}$ ). Accordingly, no fluorescence is observed. The triplet excited benzophenone may disappear in the energy dissipative processes (1): the first-order phosphorescence (1a) and ISC (1b), as well as the second-order triplet-triplet annihilation reaction (1c). The primary photoreduction step (2) produces an equal amount of the aliphatic and aromatic ketyl radicals, which disappear in the second order self- (3 and 6) and cross termination (4) reactions. Reaction (6) is predominantly a combination process; (3) and (4) have several product channels. ${ }^{16}$ In this study our interest is focused on the kinetic features of the photoreduction processes, in particular the secondary step (5). Thus only the reactant sides of the radical - radical termination reactions are considered in the mechanism. 
In the first step the spectroscopic properties of the new derivatives were measured; $n$-hexane and acetonitrile were used as aprotic apolar and polar solvents, respectively. Studies in the former solvent allow us to characterize the substituent effect on hydrogen bond complexation of benzophenone derivatives with isopropanol and, for more expressed effects, with hexafluoroisopropanol (HFIP). In the reaction kinetic experiments, acetonitrile was chosen as an almost ideal solvent. The primary photoreduction step (reaction 2) was studied separately from the other processes via measuring the phosphorescence decay rates.

Our interest is focused on reaction (5), the secondary reduction of the aromatic ketone by aliphatic ketyl radicals formed in the primary hydrogen atom transfer. The rate coefficient of reaction (5) was determined in a two-step procedure. First we measured the rate of the radicalradical reaction (4) at high light intensity and low ketone concentration by laser flash photolysis - transient absorption, then the conditions were changed to values more optimal for the determination the rate of the radical conversion process (5), i.e. at high ketone concentration and at low excitation light intensity. We explored the dependence of the observable rate coefficient for reaction (5) on the alcohol concentration of the solvent, from which we expect to learn about the role of hydrogen-bonded complexes in the reaction. For additional information on the reaction, and to understand how the newly prepared derivatives can be used in photochemical kinetic studies, we determined the rate coefficient of the photoreduction by an agent with reduced hydrogen atom donor capability, gamma-valerolactone. The experimental studies of spectroscopic properties and the kinetic processes were supplemented by electronic structure theory calculations.

In the rest of the paper we first present the spectroscopic and photophysical properties of the benzophenone derivatives including experimental and electronic structure data, followed by the 
results on the rates of the primary and secondary photoreduction reactions. Finally we analyze the effect of the polarity of solvent and the nature of the reactant on the kinetic features of the system.

\section{EXPERIMENTAL AND THEORETICAL METHODS}

Experiments. The absorption spectra were recorded on a thermoregulated Unicam UV500 spectrophotometer with a resolution typically of $0.5 \mathrm{~nm}$. When necessary, correction was made for dilution caused by the addition of alcohol and for density change due to variation in temperature. The corrected phosphorescence spectra were obtained on a Jobin-Yvon Fluoromax photon counting equipment with $0.5 \mathrm{~nm}$ resolution, as well as on a quantum corrected Shimadzu RF-5000PC spectrofluorimeter. Excitation wavelength was in the range of 330-366 nm. The calibration of the wavelength scale was made just prior to the measurements with a medium pressure mercury arc comparing the measured data with the values of the NIST data base. ${ }^{18}$ Room temperature phosphorescence quantum yields were determined relative to that of quinine sulfate in $1 n \mathrm{H}_{2} \mathrm{SO}_{4}\left(\Phi_{\mathrm{f}}=0.546\right) .{ }^{19}$ In all measurements (except some absorption ones) freezepump-thaw degassed samples were used in 1x1x4 cm Suprasil quartz cells.

The triplet yields $\left(\Phi_{\mathrm{isc}}\right)$ of the new benzophenone derivatives were measured by laser flash photolysis using the energy transfer method with perylene as energy acceptor. ${ }^{20}$ The excitation wavelength was $308 \mathrm{~nm}$ from a $\mathrm{XeCl}$ exciplex laser (Lambda Physik Compex), or $355 \mathrm{~nm}$ from a frequency-tripled Nd:YAG laser (Continuum Surelight). The measurements were made relative

to the triplet yield of benzophenone in acetonitrile $\left(\Phi_{\mathrm{isc}}=1.00\right) .{ }^{16 \mathrm{~b}, 21}$ The molar extinction coefficients of the different transient species were determined relative to that of benzophenone triplet $\left(6600 \pm 100 \mathrm{~mol}^{-1} \mathrm{dm}^{3} \mathrm{~cm}^{-1} \text { at } 525 \mathrm{~nm}\right)^{16 \mathrm{~b}}$ measured at the same optical density (around 0.5 ) in acetonitrile at $22{ }^{\circ} \mathrm{C}$. The repetition rate of the excitation in the transient absorption 
measurement was less than a quarter of a Hertz, to avoid the bias of the signal at higher pulse frequency.

\section{Scheme 2.}<smiles>O=C(c1cc(C(F)(F)F)cc(C(F)(F)F)c1)c1cc(C(F)(F)F)cc(C(F)(F)F)c1</smiles><smiles>[R]c1ccc(C(=O)c2ccc([R])cc2)cc1</smiles>

3,3',5,5'-tetrakis(trifluoromethyl)benzophenone (Bis[3,5-bis(trifluoromethyl)phenyl]methanone, CFBp), 4,4'-di(trifluoromethyl)benzophenone (dpCFBp, $\mathrm{R}=\mathrm{CF}_{3}$ ) and 4,4'-dimethoxybenzophenone $\left(\mathrm{MeOBp}, \mathrm{R}=\mathrm{CH}_{3} \mathrm{O}\right)$

Benzophenone (Bp) and 4,4'-dimethoxybenzophenone (MeOBp) was received from Aldrich and were recrystallized from hexane, while 4,4'-di(trifluoromethyl)benzophenone (dpCFBp) and 3,3',5,5'-tetrakis(trifluoromethyl)-benzophenone $\{$ bis[3,5-bis(trifluoromethyl)phenyl]methanone, CFBp $\}$ was synthesized for this study from ethyl formate and from the corresponding aril bromide. The detailed description of preparation, purification and characterization of the compounds can be found in the Supporting Information. The n-hexane, acetonitrile (MeCN), and 2-propanol (IPA) were of Merck Uvasol quality, while the fluorinated alcohol (i.e. 1,1,1,3,3,3-hexafluoropropan-2-ol (HFIP)) were purchased from Fluorochem Ltd. and were used without further purification. $\gamma$-Valerolactone was Sigma-Aldrich, while other solvents, perfluoromethylcyclohexane (pfmch), paraffin, benzene, EPA components (n-pentane, diethyl ether and ethanol), triacetin, and butyronitrile ( $\operatorname{PrCN})$ were received from Merck, and purified on a combined activated charcoal - alumina column before use. 
Electronic structure calculations. The structures and energies of the species participating in the secondary photoreduction of the four benzophenone derivatives were studied by density functional theory (DFT) using the $\mathrm{B} 3 \mathrm{LYP}^{22}$ and $\mathrm{BB} 1 \mathrm{~K}^{23}$ combination of functionals. The species studied are: ketones, ketyl radicals, van der Waals complexes formed by ketones and ketyl radicals, as well as the transition structures corresponding to the $\mathrm{H}$ atom transfer reaction (5). The structures were fully optimized. Due to the large charges in the $\mathrm{CF}_{3^{-}}$and methoxysubstituted structures, there are numerous van der Waals minima on the potential surfaces of the bimolecular complexes involving such species, corresponding to several conformations involving hydrogen bonding. When reporting data on van der Waals complexes, the lowestenergy structures we found are considered. The transition structures corresponding to the saddle point of the PES of the H-transfer reactions are characterized by a well-defined imaginary frequency which shows clearly that the motion involved in the reaction is mostly the breakage of one $\mathrm{O}-\mathrm{H}$ or $\mathrm{C}-\mathrm{H}$ bond and formation of a new $\mathrm{H}-\mathrm{O}$ bond. In the calculations the B3LYP functionals were combined with the $6-31 G^{*}$ basis set for exploratory calculations and the BB1K functional with the cc-pVDZ basis set to get more accurate energies and geometries. The substituent effect on the absorption spectra of the ketone was studied by getting the singlet excited state energy by time-dependent $\mathrm{DFT}^{24}$ at the equilibrium geometry of the ground state of each ketone. The calculations were performed using the Gaussian 03 suite of programs.

\section{RESULTS}

\section{The absorption and phosphorescence spectra of ring-substituted benzophenone derivatives}

The absorption spectra of the four examined compounds in $n$-hexane are shown in Figure 1. Here only the shape of the lowest $n-\pi^{*}$ transition is given together with an approximate vibronic resolution; the full spectra can be seen in Figure S1 of the Supporting Information. The position 
of the $0-0$ transition is the measure of the energy of the singlet excited state at the ground state geometry. Both the structural and solvent relaxation are expected to be small for the excited benzophenone derivatives,${ }^{25}$ especially in the apolar $n$-hexane. The energy of the $0-0$ transitions of the absorption spectrum (see Table 1) decreases with the increase of the electron withdrawing ability of the substituent on the phenyl group, the gap being the lowest for dpCFBp, where the trifluoromethyl groups are in the para position.

Aromatic ketones can form hydrogen-bonded complexes with alcohols, the proton acceptor being the $\mathrm{C}=\mathrm{O}$ group which is also the chromophore. The equilibrium constant $\left(K_{\text {eq }}\right)$ and the concomitant spectral changes can shed light on the influence of the ring substituent on the chromophore in the ketone. To investigate this effect, we selected HFIP as a very strong hydrogen bond donor at $298 \mathrm{~K}$ in $n$-hexane and determined $K_{\text {eq }}$ and the spectra of the complexes (with the methodology we used before for the HFIP - 4-(dimethylamino)pyridine system ${ }^{26}$ ). The equilibrium constants for the formation of a H-bonded complex between HFIP and the four benzophenone derivatives were determined from the alcohol concentration dependence of the spectra and are shown in Table $1 . K_{\text {eq }}$ gradually increases with increasing electron donor quality of the substituent of the aromatic ring: $\ln \left(\mathrm{K}_{\mathrm{eq}}\right)$ changes linearly with the Hammett constant of the substituent. The absorption spectra (shown in Figure 1) shift to the blue when the complex is formed, at the same time the oscillator strength increases as it has been seen before with other molecules. ${ }^{20}$ Upon complexation, the singlet energy increases by approximately $12 \mathrm{~kJ} \mathrm{~mol}^{-1}$ for all compounds. The hypsochromic shift observed in the absorption spectrum of the $n-\pi^{*}$ excitation of a carbonyl molecule is due to the reduction of the electron density on the oxygen atom with excitation. 
Table 1. Selected Photophysical and Spectroscopic Parameters of the Four Benzophenone Derivatives.

\begin{tabular}{|c|c|c|c|c|c|}
\hline \multicolumn{2}{|c|}{ Compounds: } & CFBp & dpCFBp & Bp & MeOBp \\
\hline \multicolumn{6}{|c|}{$n$-hexane } \\
\hline \multicolumn{2}{|c|}{${ }^{1} \boldsymbol{E}(0-0) / \mathrm{cm}^{-1}$} & 26075 & 25940 & 26435 & 27005 \\
\hline \multicolumn{2}{|c|}{${ }^{1} \boldsymbol{E} / \mathrm{kJ} \mathrm{mol}^{-1 \mathrm{~b}}$} & 309.5 & 308.5 & $\begin{array}{c}314 \\
(311)^{d}\end{array}$ & 321 \\
\hline \multirow{2}{*}{${ }^{1} \boldsymbol{v}_{\mathrm{abs}}(\max ) / \mathrm{nm}$} & meas. & 350.5 & 352.5 & 347.0 & $328.0^{f}$ \\
\hline & calc. & 347.7 & 352.2 & 346.1 & 339.4 \\
\hline \multicolumn{2}{|c|}{${ }^{1} \boldsymbol{\varepsilon}_{\mathrm{abs}}(\max ) / \mathrm{dm}^{3} \mathrm{~mol}^{-1} \mathrm{~cm}^{-1}$} & 101.3 & 99.0 & 103.0 & 335 \\
\hline \multirow{2}{*}{$\left|{ }^{1} \boldsymbol{M}\right|_{\mathbf{g} \rightarrow \mathbf{e}} /$ Debye } & meas. & 0.355 & 0.345 & 0.347 & 0.59 \\
\hline & calc. & 0.33 & 0.32 & 0.31 & 0.49 \\
\hline \multicolumn{2}{|c|}{$\boldsymbol{K}_{\mathrm{eq}} / \mathrm{dm}^{3} \mathrm{~mol}^{-1 \mathrm{a}}$} & 1.9 & 11.2 & 40 & 140 \\
\hline \multicolumn{2}{|c|}{${ }^{\mathbf{3}} \boldsymbol{E} / \mathrm{kJ} \mathrm{mol}^{-1 \mathrm{~b}}$} & 283.5 & $\begin{array}{l}\mathbf{2 8 0 . 5} \\
(276)^{\mathrm{d}}\end{array}$ & $\begin{array}{c}\mathbf{2 8 6 . 5} \\
(287)^{\mathrm{d}} \\
(287.7 \pm 0.4)^{\mathrm{e}}\end{array}$ & $\begin{array}{c}\mathbf{2 8 9 . 5} \\
(293)^{\mathrm{d}} \\
(288.9 \pm 0.8)^{\mathrm{e}}\end{array}$ \\
\hline \multicolumn{2}{|c|}{${ }^{3} \boldsymbol{E}_{\text {calc }} / \mathrm{kJ} \mathrm{mol}^{-1}$} & 261.3 & 258.8 & 266.4 & 275.6 \\
\hline \multicolumn{6}{|c|}{$\mathrm{MeCN}$} \\
\hline \multicolumn{2}{|c|}{${ }^{1} \boldsymbol{E} / \mathrm{kJ} \mathrm{mol}^{-1 \mathrm{~b}}$} & 316.5 & 313 & $\begin{array}{c}319 \\
(316)^{\mathrm{d}}\end{array}$ & $\begin{array}{c}325 \\
(328)^{d}\end{array}$ \\
\hline \multirow{2}{*}{${ }^{1} \boldsymbol{v}_{\mathrm{abs}}(\max ) / \mathrm{nm}$} & meas. & 341.7 & 345.5 & 339.0 & 332.3 ${ }^{\mathrm{g}}$ \\
\hline & calc. & 339.5 & 346.4 & 337.0 & 330.3 \\
\hline \multicolumn{2}{|c|}{${ }^{1} \varepsilon_{\mathrm{abs}}(\max ) / \mathrm{dm}^{3} \mathrm{~mol}^{-1} \mathrm{~cm}^{-1}$} & 105.8 & 103.5 & 141.0 & \\
\hline \multirow{2}{*}{$\left|{ }^{1} \boldsymbol{M}\right|_{\mathbf{g} \rightarrow \mathbf{e}} /$ Debye } & meas. & 0.34 & 0.35 & 0.385 & $0.7^{\mathrm{g}}$ \\
\hline & calc. & 0.34 & 0.31 & 0.31 & 0.55 \\
\hline \multicolumn{2}{|c|}{${ }^{3} \boldsymbol{E} / \mathrm{kJ} \mathrm{mol}^{-1 \mathrm{~b}, \mathrm{c}}$} & 284.5 & 281.5 & $\begin{array}{l}\mathbf{2 8 9 . 5} \\
(289)^{d}\end{array}$ & $\begin{array}{l}\mathbf{2 9 2 . 5} \\
(293)^{d}\end{array}$ \\
\hline \multicolumn{2}{|l|}{$\boldsymbol{\Phi}_{\mathrm{ph}}$} & 0.004 & 0.005 & 0.006 & 0.007 \\
\hline \multicolumn{2}{|l|}{${ }^{3} \tau_{0} / \mu s$} & 28 & 20 & 36 & 86 \\
\hline \multicolumn{2}{|c|}{$\boldsymbol{k}_{\mathrm{ph}} / \mathrm{s}^{-1}$} & 145 & 240 & 170 & 86 \\
\hline \multirow{2}{*}{\multicolumn{2}{|c|}{\begin{tabular}{c|c}
$\left.{ }^{3} \boldsymbol{M}\right|_{\mathbf{t r} \rightarrow \mathbf{g}} /$ Debye \\
${ }^{3} \boldsymbol{v}^{\max }(\mathrm{TT}) / \mathrm{nm}$
\end{tabular}}} & 0.0051 & 0.0067 & 0.0053 & 0.0037 \\
\hline & & 537 & 540 & $525^{\text {h }}$ & 547 \\
\hline \multicolumn{2}{|c|}{${ }^{3} \boldsymbol{\varepsilon}^{\max }(\mathbf{T T}) / \mathrm{dm}^{3} \mathrm{~mol}^{-1} \mathrm{~cm}^{-1}$} & 6350 & 7100 & $6600^{h}$ & 6870 \\
\hline \multicolumn{2}{|c|}{$\boldsymbol{v}^{\max }($ ketyl $) / \mathrm{nm}$} & 564 & 565 & $540^{\mathrm{h}}$ & 560 \\
\hline \multicolumn{2}{|c|}{$\boldsymbol{\varepsilon}^{\max }\left(\right.$ ketyl) $/ \mathrm{dm}^{3} \mathrm{~mol}^{-1} \mathrm{~cm}^{-1}$} & 4880 & 5800 & $3540^{h}$ & 3140 \\
\hline
\end{tabular}

)$^{\mathrm{a}}$ with HFIP, $)^{\mathrm{b}}$ corrected for Stokes shift, see text, $)^{\mathrm{c}}$ Triplet energy at $-45^{\circ} \mathrm{C}$, $\left.)^{\mathrm{d}} \operatorname{Ref} 21 \mathrm{a},\right)^{\mathrm{e}}$ Ref $21 \mathrm{~b}$ in ethyl acetate, $)^{\mathrm{f}}$ different vibronic band, see text, $)^{\mathrm{g}}$ estimated from the calculated wavelength by the theory-experiment shift for Bp, see text, , $)^{\mathrm{h}}$ Ref. $16 \mathrm{~b}$ 
The complete absorption spectra are shown in Figure S1 (Supporting Information) both in $n$ hexane and in the polar acetonitrile solution. The ordering of the energy of the intense $\pi-\pi^{*}$ transitions are more or less opposite to what is observed for the lowest excited $n-\pi^{*}$ state, in case of MeOBp the separation becomes relatively low. With increasing polarity of the solvent - as it is expected for an $n-\pi^{*}$ transition - the energy of the first singlet state increases by about $6 \mathrm{~kJ}$ $\mathrm{mol}^{-1}$ (cca. $8 \mathrm{~nm}$ ), while the energy of the higher $\pi-\pi^{*}$ states (corresponding to a symmetry allowed transitions) decreases.

The transition dipole moments were derived as described in the paper of Lewis and Maroncelli ${ }^{27}$ (using Bakhshiev's effective field correction) terminating the integration at the minimum of the absorption curve. The transition dipole moment of the $n-\pi^{*}$ absorption band is almost independent of the nature of the substituents and the solvent polarity as well (Table 1); significant deviation from the others can be seen only in case of MeOBp. (It should be noted that the uncertainty of the determination of $\left|{ }^{1} M\right|_{\mathrm{g} \rightarrow \mathrm{e}}$ of MeOBp in acetonitrile is much larger than the corresponding data for the other molecules, partly because here no minimum can be found on the absorption curve, allowing only an approximate separation of the $n-\pi^{*}$ and $\pi$ - $\pi^{*}$ bands.) The phosphorescence spectra of the four benzophenone derivatives are depicted in Figure 2. The shapes are similar for all compounds, and the ordering of the energy of the triplet state (from the vibrationally resolved 0-0 band) is the same as seen for the singlet energy. The phosphorescence quantum yield and the lifetime of the triplet state depend very much on the purity of the acetonitrile solvent as well as on the circumstances of sample preparation. For example, the minor LAT photoproduct (the "light-absorbing-transient", a long lived transient combination product of the reaction (4), resembling Gomberg's hydrocarbon) quenches the triplet with a diffusion controlled rate. ${ }^{16 \mathrm{~b}}$ Consequently, the relevant data are the phosphorescence rate 
coefficients $\left(k_{\mathrm{ph}}\right)$, or even more the corresponding transition dipole moments (see Table 1). These properties do not show any remarkable influence of substitution and are similar to what was published before for other benzophenone derivatives. ${ }^{28}$

The triplet state energies (corresponding to the triplet vs. ground state energy difference at the excited state geometry) derived from the maximum of the deconvoluted 0-0 vibronic band of the phosphorescence spectra are given in Table 2. The influence of the environment is small, as it is expected when the dipole moment change is small in the transition. ${ }^{25}$ However, it is well observable that the data determined in EPA glass are larger than expected from the polarity of the solvent mixture.

Table 2. The Emission Energy of the Triplet Benzophenone Derivatives Derived from the Maximum of the Deconvoluted 0-0 Phosphorescence Band under Different Conditions.

\begin{tabular}{|c|c|c|c|c|c|}
\hline Solvent & Temperature & \multicolumn{5}{|c|}{ Energy of the 0-0 emission of the triplet $/ \mathrm{cm}^{-1}$} \\
\cline { 3 - 6 } & ${ }^{\circ} \mathrm{C}$ & MeOBp & Bp & dpCFBp & CFBp \\
\hline pfmch $^{\mathrm{a}}$ & 25 & $--^{\mathrm{b}}$ & 23800 & 23300 & 23515 \\
\hline$n$-hexane & -90 & 24022 & 23771 & $--^{\mathrm{c}}$ & $--^{\mathrm{c}}$ \\
\hline paraffin & -100 & 24198 & 23833 & 23375 & 23613 \\
\hline benzene & 25 & 24010 & 23815 & $--^{\mathrm{c}}$ & $--^{\mathrm{c}}$ \\
\hline triacetin & -50 & 24050 & 23820 & 23255 & 23540 \\
\hline acetonitrile & 25 & 24097 & 23890 & 23280 & 23525 \\
\hline acetonitrile & -45 & 24198 & 23938 & 23270 & 23525 \\
\hline butyronitrile & -140 & 24214 & 23998 & 23280 & 23583 \\
\hline EPA & -192 & 24423 & 24194 & 23710 & 24200 \\
\hline
\end{tabular}

)$^{\mathrm{a}}$ perfluoromethylcyclohexane, $)^{\mathrm{b}}$ insoluble, $)^{\mathrm{c}}$ no signal

\section{Absorption spectra and quantum yields of the short lived transient species}

For the characterization of a kinetic system, the first step is the description of the transient species: the triplet excited Bp derivatives, the ketyl radicals formed from them via hydrogen atom abstraction from the alcohol. 
Triplet benzophenone in acetonitrile is one of the most studied and best known photochemical transients. $^{3,16}$ The formation yield of 1.00 is well established ${ }^{16 b, 21}$ and widely accepted. The molar absorption coefficient of $6600 \mathrm{~mol}^{-1} \mathrm{dm}^{3} \mathrm{~cm}^{-1}$ at the $525 \mathrm{~nm}$ maximum of the triplet-triplet (TT) absorption spectrum is the most universally used standard value in the relative determination of molar absorbance. ${ }^{16 \mathrm{~b}, 21 \mathrm{a}}$ In this study as well, the parameters of the other benzophenone derivatives were determined relative to the corresponding data $\left(\Phi_{\mathrm{ISC}},{ }^{3} \varepsilon^{\max }\right)$ of unsubstituted benzophenone.

The triplet quantum yields were determined in $\mathrm{MeCN}$ with the energy transfer method using perylene as energy acceptor and Bp as reference. For all three Bp derivatives the triplet yield was found to be unity within the experimental uncertainties $(1.01 \pm 0.02,0.99 \pm 0.02$ and $1.01 \pm 0.02$ for CFBp, dpCFBp and MeOBp, ${ }^{21 a}$ respectively). The transient absorption spectra of the aromatic ketyl radicals were recorded in acetonitrile solution containing $1.306 \mathrm{~mol} \mathrm{dm}^{-3}(10 \mathrm{v} \%)$ propan-2ol after the very quick decay of the triplet. When deriving the absorption coefficients, the formation yield of the aromatic ketyl radical was taken to be 1.00. The absorption of the aliphatic ketyl radical formed in the same reaction is negligible in the examined spectral range. ${ }^{16}$ The spectrum of the ketyl radical of MeOBp was measured in neat IPA as solvent, where the competition between the reactive and nonreactive decay channels of triplet was more favorable for ketyl formation than in acetonitrile diluted solvent mixture.

The spectra of the radical anions, the de-protonated forms of the aromatic ketyl radicals were also recorded (in the presence of $0.1 \mathrm{~mol} \mathrm{dm}^{-3} \mathrm{DABCO}$, see Figure 3) for comparative purposes. The spectra of the three types of short-lived species for the four derivatives are shown in Figure 3 (the absorption curves of the triplet ketone and of the ketyl radicals of unsubstituted benzophenone are from our earlier work ${ }^{16 a}$ ). The features of the spectra of the transients formed 
from substituted $\mathrm{Bp}$ derivatives are similar to those of the corresponding species derived from benzophenone itself with some moderate shift. The only exception is the triplet-triplet absorption spectrum of MeOBp, where a strong peak can be seen at about $430 \mathrm{~nm}$ and the absorbance remains relatively strong above $600 \mathrm{~nm}$ (see below).

\section{The primary photoreduction of the benzophenone derivatives by 2-propanol in acetonitrile}

The most conspicuous feature of the photochemistry of triplet benzophenone derivatives is that the phosphorescence decay gives us an accurate, easy-to-use tool to follow the kinetics of the primary photoreduction. Based on Scheme 1, at low light intensity the phosphorescence decay is expected to be single exponential, and the reciprocal lifetime (the sum of the first order rate coefficients of channels (1a), (1b) and the pseudo first order $k_{2}^{\prime}=k_{2}$ [IPA] of channel 2) to depend linearly on the quencher concentration. This is what we observe with laser energy $\leq 0.1$ $\mathrm{mJ})$. The slope of $1 / \tau$ vs. [IPA] line (Figure S2 showing, as an example, the relevant data on the CFBp - IPA - MeCN system) gives us the value of the $k_{2}$ rate coefficient. We also derived the Arrhenius parameters $A_{2}$ and $E_{2}$ for the four derivatives were obtained from measurements of $k_{2}$ between -50 and $85^{\circ} \mathrm{C}$ (Figure 4 ).

As it is demonstrated in Table 3, the substitution of the aromatic rings induces a remarkable change in the reactivity of triplet benzophenone towards the alcohol (the primary photoreduction step). Inspection of the Arrhenius parameters shows that the preexponential factors are very close to the expected value of $10^{8} \mathrm{dm}^{3} \mathrm{~mol}^{-1} \mathrm{~s}^{-1}$ for all four derivatives ${ }^{12}$, and the difference in reactivity is due to the monotonous change of the activation energy with the Hammett parameter of the substituent. 
Table 3. The $298 \mathrm{~K}$ rate coefficients and the Arrhenius parameters of the primary photoreduction reaction (2) in acetonitrile as well as the secondary photoreduction step (5), the radical - radical reactions (4) and (6) in $1.31 \mathrm{~mol} \mathrm{dm}^{-3} \mathrm{IPA}-\mathrm{MeCN}$ mixture

\begin{tabular}{|l|c|c|c|c|}
\hline Compound: & CFBp & dpCFBp & Bp & MeOBp \\
\hline $\boldsymbol{k}_{2} / \mathrm{dm}^{3} \mathrm{~mol}^{-1} \mathrm{~s}^{-1}$ & $(5.44 \pm 0.11) \times 10^{7}$ & $(1.45 \pm 0.05) \times 10^{7}$ & $(2.3 \pm 0.3) \times 10^{6}$ & $(2.83 \pm 0.15) \times 10^{5}$ \\
\hline $\lg \left(\boldsymbol{A}_{2} / \mathrm{dm}^{3} \mathrm{~mol}^{-1} \mathrm{~s}^{-1}\right)$ & $8.30 \pm 0.02$ & $8.03 \pm 0.07$ & $7.61 \pm 0.05$ & $7.57 \pm 0.07$ \\
\hline $\boldsymbol{E}_{2} / \mathrm{kJ} \mathrm{mol}^{-1}$ & $3.18 \pm 0.07$ & $5.18 \pm 0.36$ & $7.03 \pm 0.28$ & $12.54 \pm 0.41$ \\
\hline & & & & \\
\hline $\boldsymbol{k}_{4} / \mathrm{dm}^{3} \mathrm{~mol}^{-1} \mathrm{~s}^{-1}$ & $6.4 \times 10^{9}$ & $3.2 \times 10^{9}$ & $(8.0 \pm 0.4) \times 10^{8}$ & $(8.9 \pm 0.7) \times 10^{8}$ \\
\hline $\lg \left(\boldsymbol{A}_{4} / \mathrm{dm}^{3} \mathrm{~mol}^{-1} \mathrm{~s}^{-1}\right)$ & $10.60 \pm 0.3$ & $10.22 \pm 0.23$ & $10.14 \pm 0.15$ & $10.7 \pm 0.2$ \\
\hline $\boldsymbol{E}_{4} / \mathrm{kJ} \mathrm{mol}^{-1}$ & $4.5 \pm 1.5$ & $4.1 \pm 1.3$ & $7.1 \pm 0.8$ & $10.4 \pm 0.7$ \\
\hline & & & & \\
\hline $\boldsymbol{k}_{5} / \mathrm{dm}^{3} \mathrm{~mol}^{-1} \mathrm{~s}^{-1}$ & $(2.20 \pm 0.34) \times 10^{7}$ & $(3.15 \pm 0.85) \times 10^{6}$ & $(4.67 \pm 0.21) \times 10^{5}$ & $(2.02 \pm 0.17) \times 10^{5}$ \\
\hline $\lg \left(\boldsymbol{A}_{5} / \mathrm{dm}^{3} \mathrm{~mol}^{-1} \mathrm{~s}^{-1}\right)$ & $8.09 \pm 0.13$ & $7.94 \pm 0.22$ & $7.84 \pm 0.10$ & $8.22 \pm 0.16$ \\
\hline $\boldsymbol{E}_{5} / \mathrm{kJ} \mathrm{mol}^{-1}$ & $4.3 \pm 0.7$ & $8.2 \pm 1.2$ & $12.4 \pm 0.7$ & $16.6 \pm 0.9$ \\
\hline & & & & \\
\hline $\boldsymbol{k}_{6} / \mathrm{dm}^{3} \mathrm{~mol}^{-1} \mathrm{~s}^{-1}$ & $3.8 \times 10^{8}$ & $(3.8 \pm 1.1) \times 10^{8}$ & $(5.9 \pm 0.1) \times 10^{7}$ & $(1.5 \pm 0.2) \times 10^{8}$ \\
\hline $\lg \left(\boldsymbol{A}_{6} / \mathrm{dm}^{3} \mathrm{~mol}^{-1} \mathrm{~s}^{-1}\right)$ & $9.65 \pm 0.3$ & $9.5 \pm 0.2$ & $10.05 \pm 0.25$ & $10.4 \pm 0.2$ \\
\hline $\boldsymbol{E}_{6} / \mathrm{kJ} \mathrm{mol}^{-1}$ & $6.0 \pm 1.8$ & $5.1 \pm 1.1$ & $13.1 \pm 1.4$ & $13.0 \pm 0.9$ \\
\hline
\end{tabular}

\section{The reactions involving the aromatic ketyl radicals}

In our earlier study ${ }^{13}$ it was shown that at very low excitation light intensity, the rate coefficient of the radical transfer reaction (5) can be reliably determined in direct measurements by monitoring of the decay of the absorption of the aromatic ketyl radical if relatively high ketone concentration is used. When the benzophenone concentration is large, the metathesis reaction (5) \{being in the pseudo first order regime with $k_{5}{ }^{\prime}=k_{5}[$ ketone] $\}$ is the dominant process consuming the dimethyl ketyl radical, because the relative influence of the second order radical - radical reactions is suppressed by the purposeful reduction of the excitation light intensity. Moreover, at low light intensity the uncertainty of the determination of $k_{5}$ is smaller than at larger intensities 
when the radical-radical reactions need to be taken into account when modeling the decay curves and the error of their rate constants is propagated into that of $k 5$.

At normal laser light intensities $(50-100 \mathrm{~mJ} / \mathrm{flash})$ when the concentration of the formed aromatic ketyl radical is larger, its transient absorption (monitored at $540 \mathrm{~nm}$ ) decays monotonously. ${ }^{13,16 a}$ The decay of the absorption signal at the beginning is relatively quick, because not only reactions (5) and (6) but also the more efficient reaction (4) consume the aromatic radicals. After the aliphatic radicals are completely consumed in reactions (3), (4) and (5), the decay of the aromatic ketyl radicals becomes strictly second order. The corresponding set of differential equations cannot be integrated analytically. Instead, the decay curves were simulated by numerical integration and the rate constants $k_{4}, k_{5}$ and $k_{6}$ were optimized to fit the calculated time resolved transient signal to the measured. As it was shown before, ${ }^{16 a}$ it is essential to keep the rate parameter of reaction (3) fixed in the fitting procedure. Fortunately, $k_{3}$ can be determined directly in the acetone - 2-propanol - acetonitrile system at the same solvent composition as the target system. ${ }^{12,16 a, 29}$

The rate parameters of the radical - radical reactions were determined separately to minimize the uncertainty of the determination of the rate coefficient of reaction (5). The characteristic increase of the rate coefficients of radical - radical reactions (4) and (6) observed when the reacting ketyl radicals are substituted with electron-withdrawing groups is due to the decrease of the activation energy (see Table 3).

The reliability of the measured values of $k_{6}$ and its increase with tetra-meta $\mathrm{CF}_{3}$ substitution was checked in direct transient absorption measurements in two systems under conditions when no cross reactions (4) are possible. The second-order decay of the aromatic ketyl radical was 
monitored in the Bp - benzhydrol - acetonitrile and in the CFBp tetra(trifluoromethyl)benzhydrol (i.e. bis[3,5-bis(trifluoromethyl)-phenyl]methanol, CFBH) acetonitrile system, where in the primary photoreduction step both the ketone and the alcohol yields the same aromatic ketyl radical. The derived $k_{6}$ values are $5.9 \times 10^{7}$ and $3.8 \times 10^{8} \mathrm{dm}^{3} \mathrm{~mol}^{-1} \mathrm{~s}^{-}$ ${ }^{1}$, respectively, in excellent agreement with those derived from the photoreduction experiments by IPA. It should be mentioned that in the photoreaction of CFBp with CFBH (in contrast to Bp with benzhydrol), the absorbance was found to increase with the duration of irradiation in the 290-350 nm range. The absorbance decreased when the sample was saturated by air, indicating the formation and loss of an oxygen-sensitive, long-lived intermediate photoproduct characterized by maximum absorption at $318 \mathrm{~nm}$.

CFBp and dpCFBp show the typical features of secondary ketyl radical formation even at high laser light intensity (Figure 5a), contrary to $\mathrm{Bp}$ and $\mathrm{MeOBp}$ for which the secondary increase in the time resolved signal of ketyl radical absorption can be observed only at low excitation light intensity and at relatively large ketone concentration. When the light intensity is reduced, the kinetics of the secondary formation of the absorbing species exhibits an almost perfectly exponential build-up (pseudo first order formation process of the aromatic ketyl, Figure 5b). Plotting the derived pseudo first order rate coefficient, $k_{5} \square=k_{5}$ [ketone] as a function of the ketone concentration (see as an example, the plot for CFBp in Figure S3 of Supporting Material) the second-order rate coefficient $k_{5}$ can be easily derived. The Arrhenius parameters of the radical transfer reactions were determined by repeating the procedure at different temperatures and considering also that the concentration of the reactant is changing with the change of solvent density (Table 3 and Figure 6). The influence of the aryl substitution - similarly to the rate constant of the primary step (2) - is remarkable: there is an almost two order of magnitude 
difference between the room temperature values of $k_{5}$ of dimethoxy- and the tetra(trifluoromethyl) derivatives. The increase of reactivity with the electron-withdrawing nature of the substituent is due to the decrease of the activation energy, since the preexponential factors are all around $10^{8} \mathrm{dm}^{3} \mathrm{~mol}^{-1} \mathrm{~s}^{-1}$ (what it is expected for a hydrogen transfer process). ${ }^{12}$

In Figure 5b, the transient absorption curves measured at $565 \mathrm{~nm}$ and $810 \mathrm{~nm}$ in the CFBp - 2propanol - acetonitrile system are shown. The detection wavelengths correspond to the absorption maxima of ketyl radical and the radical anion, respectively (see Figure 3a). The shape of the build-up curves is similar, the ratio of the absorbance is constant, around 45, indicating that the both signals belong to the ketyl radical and that anion radical formation, if any, is negligible (see below in the discussion). From this, one can conclude that in neutral solution direct electron transfer does not have a significant role in the photoreduction of benzophenone derivatives.

\section{The effect of solvent composition and polarity on the hydrogen atom transfer rate} coefficients $k_{2}$ and $k_{5}$

The discrepancy of $k_{5}$ for photoreduction of Bp measured in 1.31 and $2.62 \mathrm{~mol} \mathrm{dm}^{-3}$ 2-propanol in acetonitrile indicates that the solvent composition influences the rate of the reaction (data at the latter concentration are from Ref. 13). To get a clearer picture, $k_{2}$ and $k_{5}$ were determined in benzene, an apolar solvent in which the effect of hydrogen bond interactions is expected to be stronger than in acetonitrile. The Arrhenius plots of the rate constants of the two reduction steps, reaction (2) and (5) are shown in Figure 4S in the Supporting Material. The rate parameters of the primary photoreduction process $(2)$ are very similar $\left\{k_{2}\left(25^{\circ} \mathrm{C}\right)=2.2 \times 10^{6} \mathrm{dm}^{3} \mathrm{~mol}^{-1} \mathrm{~s}^{-1}, \lg \left(A_{2} /\right.\right.$ $\left.\left.\mathrm{dm}^{3} \mathrm{~mol}^{-1} \mathrm{~s}^{-1}\right)=7.8, E_{2}=8.5 \mathrm{~kJ} \mathrm{~mol}^{-1}\right\}$ to those measured in acetonitrile (Table 3). In contrast, for 
the metathesis reaction (5) an almost sevenfold increase can be observed in the roomtemperature rate coefficient $\left\{k_{5}\left(25{ }^{\circ} \mathrm{C}\right)=(3.45 \pm 0.42) \times 10^{6} \mathrm{dm}^{3} \mathrm{~mol}^{-1} \mathrm{~s}^{-1}\right\}$ in benzene, compared with the value derived in $\mathrm{MeCN}$. The increase can be attributed more to the change of the preexponential factor $\left\{\lg \left(A_{5} / \mathrm{dm}^{3} \mathrm{~mol}^{-1} \mathrm{~s}^{-1}\right)=9.0 \pm 0.2, E_{5}=(14.0 \pm 1.1) \mathrm{kJ} \mathrm{mol}^{-1}\right\}$.

The influence of the solvent composition on the primary and secondary photoreduction rate was explored in more detail in 2-propanol - acetonitrile mixtures. The mixing ratio hardly influences $k_{2}$, in agreement with the earlier observations. ${ }^{16 \mathrm{~b}}$ The room-temperature $k_{5}$, in contrast, decreases by almost a factor of two when the alcohol concentration increases (Figure 7). 


\section{Photoreduction of triplet Bp derivatives by $\gamma$-valerolacton, a slowly reacting substrate}

The relative importance of the secondary photoreduction reaction with respect to the primary step can shed light on the factors determining its rate coefficient. To study such effects, we changed the nature of the hydrogen donor. Gamma-valerolactone (GVL) was chosen as a reductant for several reasons. First, it has a relatively weakly bound $\mathrm{H}$ atom that can be abstracted by the triplet ketone. Second, the polarity of GVL is relatively large $(\varepsilon=36, n=$ 1.432), and the increase of GVL concentration does not change notably the polarity of the solvent when added in significant amounts to acetonitrile. Third, we are interested in studying the relative rate of abstraction of its different $\mathrm{H}$ atoms using deuterium labeling for which we need an appropriate reactant that reacts slowly enough to allow the distinction of various abstraction sites. This will be useful in understanding the behavior of GVL which is proposed as a "green" liquid fuel ${ }^{30}$. Here we report the observations of the primary and secondary photoreduction of Bp and CFBp by GVL.

The Arrhenius plots of rate coefficients of the primary photoreduction step by GVL as reactant are shown in Figure 8. Using triplet benzophenone as hydrogen acceptor the reaction is slow, its rate constant, $k_{\text {primary }}\left(25^{\circ} \mathrm{C}\right)=1.5 \times 10^{5} \mathrm{dm}^{3} \mathrm{~mol}^{-1} \mathrm{~s}^{-1}$, is at the limit of reliable measurements. This means that if the rate decreases further when deuterium isotope substitution is applied, it will not be accurately measurable. The enhanced reactivity of triplet CFBp promises more reliable determination of rate coefficients of various channels of photoreduction of GVL, the measured rate coefficient for $\mathrm{H}$ atom transfer being $k_{\text {primary }}\left(25^{\circ} \mathrm{C}\right)=(1.13 \pm 0.05) \times 10^{6} \mathrm{dm}^{3} \mathrm{~mol}^{-1} \mathrm{~s}^{-1}$. This is large enough to allow convenient rate measurements even at lower than $2 \%$ reactant concentration. 
Concerning the secondary photoreduction reaction, no indication was found for the occurrence of the radical transfer process analogous to reaction (5) in the CFBp - GVL - MeCN system. Furthermore, we found no example of such metathesis involving any aliphatic radical that is not able to form a hydrogen bonded complex with the ketone. This indicates that the radical-transfer reaction (5) can take place only via a hydrogen-bonded complex.

\section{Electronic structure calculations}

Our theoretical studies are focused on structural data, UV absorption spectra and the kinetics of reaction (5), the secondary photoreduction of the four benzophenone derivatives. The calculated vertical excitation energies of the first five absorption bands in vacuum, $n$-hexane and acetonitrile as well as the corresponding oscillator strengths for the four benzophenone derivatives are summarized in Table S1 in the Supporting Material. The agreement between the measured and calculated wavelength data is generally very good (the difference is considerably less than $1 \%$, see Table 1) except for MeOBp in $n$-hexane. From the experimental data shown in Figure 1 one can see that, because of the strong red shift of the intense, allowed $\pi$ - $\pi^{*}$ band, the fourth vibronic band becomes the band maximum. For the maximum of the third vibronic band $341.2 \mathrm{~nm}$ is obtained, in excellent agreement with the calculations $(339 \mathrm{~nm})$. The calculated and measured transition dipole data for the critical first band also agree well (Table 1), regarding that the calculation was made at a relatively simple level. 
Table 4. The Enthalpies of the Reactant and Product vdW Complexes, the Transition Structures and the Products of Reaction (5) with Respect to the Ketone + Dimethyl Ketyl Reactant Level (BB1K/cc-pVDZ Data, kJ mol ${ }^{-1}$ ) in Silico Vacuum and MeCN.

\begin{tabular}{|c|c|c|c|c|c|c|c|c|c|c|}
\hline Compound: & \multicolumn{2}{|c|}{ acetone } & \multicolumn{2}{c|}{ MeOBp } & \multicolumn{2}{c|}{ Bp } & \multicolumn{2}{c|}{ dpCFBp } & \multicolumn{2}{c|}{ CFBp } \\
\hline & vacuum & MeCN & vacuum & MeCN & vacuum & MeCN & vacuum & MeCN & vacuum & MeCN \\
\hline reactant complex & -29.9 & 18.86 & -30.76 & -23.27 & -26.70 & -13.18 & -24.26 & -12.26 & -20.02 & -8.60 \\
\hline$H_{\text {reac }}^{\#}(\mathrm{TS})^{\mathrm{a}}$ & 37.89 & 53.86 & 8.44 & -3.92 & 1.38 & -10.14 & -9.18 & -13.72 & -12.94 & -15.81 \\
\hline$H_{\text {compl }}^{\#}(\mathrm{TS})^{\mathrm{b}}$ & 67.79 & 72.72 & 39.20 & 19.35 & 28.09 & 3.04 & 15.08 & -1.46 & 7.07 & -7.21 \\
\hline product complex & -29.9 & -18.86 & -79.86 & -78.48 & -87.98 & -82.37 & -104.4 & -95.28 & -120.4 & -104.8 \\
\hline$\Delta H($ reac,5) & 0.0 & 0.0 & -49.15 & -59.49 & -60.50 & -65.09 & -70.92 & -77.96 & -73.21 & -79.65 \\
\hline vdWprod vs. prod. & -29.9 & -18.86 & -30.7 & -19.0 & -27.5 & -17.3 & -33.5 & -17.3 & -47.2 & -25.15 \\
\hline$\Delta H($ compl,5) & 0.0 & 0.0 & -49.1 & -55.2 & -61.3 & -69.2 & -80.1 & -83.0 & -100.4 & -96.2 \\
\hline$\Delta H^{\#}{ }_{\text {calc }}$ & & 75.46 & & 17.68 & & 11.46 & & 7.88 & & 5.79 \\
\hline$E_{2}$ from this work & & & & 12.5 & & 7.0 & & 5.2 & & 3.2 \\
\hline$E_{5}$ from this work & & & & 16.6 & & 12.4 & & 8.2 & & 4.3 \\
\hline
\end{tabular}

)$^{\mathrm{a}}$ with respect to reactants

)$^{\mathrm{b}}$ with respect to the reactant complex 
Table S2 shows selected bond lengths in singlet and triplet benzophenones and at the transition structure for reaction (5) for the four Bp derivatives and, for comparison, acetone. Table 4 summarizes the relevant enthalpies; Figure 9 presents the enthalpy profile of the reactions (5).

The O-H bond dissociation enthalpy calculated from the enthalpies of the ketones and their ketyl radicals are 132.7, 144.1, 154.5 and $156.8 \mathrm{~kJ} \mathrm{~mol}^{-1}$, respectively, for MeOBp, Bp, dpCFBp and CFBp. This indicates that the ketyl radical is stabilized by $\mathrm{CF}_{3}$ substitution by about $10 \mathrm{~kJ} \mathrm{~mol}^{-1}$, while the bis(para-methoxy) substitution destabilizes it by approximately the same amount. The effect is carried over to the enthalpy of the $\mathrm{H}$ atom transfer reaction (5) which changes from $70.9 \mathrm{~kJ} \mathrm{~mol}^{-1}$ for Bp by the same amount as the ketyl binding enthalpy.

The reactants of reaction (5), the ketyl radical of acetone and of each derivative of benzophenone form a complex held together by a hydrogen bond between the two carbonyl-type $\mathrm{O}$ atoms. The binding energy of the complex $\left(\mathrm{CH}_{3}\right)_{2} \mathrm{COH}-\mathrm{OCAr}_{2}$ (expressed as enthalpy of dissociation) is (18.9 for acetone), 23.3, 13.2, 12.1 and $8.6 \mathrm{~kJ} \mathrm{~mol}^{-1}$ for MeOBp, Bp, dpCFBp and CFBp, respectively, decreases monotonously. The products, acetone and the ketyl radical of the ketone also form a strongly bound $\left(\mathrm{CH}_{3}\right)_{2} \mathrm{CO}-\mathrm{HO} a \mathrm{Ar}_{2}$ van der Waals complex, the binding energies of which do not show a clear trend (19.0, 17.3, 17.3 and $25.1 \mathrm{~kJ} \mathrm{~mol}^{-1}$ in the same order as above). The substitution of the methyl groups of acetone in the acetone - dimethyl-ketyl radical complex by phenyl rings influences the binding energy less than the substituents of the aryl groups. The energy of the $\mathrm{O}-\mathrm{H}-\mathrm{O}$ hydrogen bond is stronger if the $\mathrm{H}$ atom is on the aliphatic carbonyl oxygen for $\mathrm{MeOBp}$, while for $\mathrm{Bp}$ and the $\mathrm{CF}_{3}$ substituted derivatives the aromatic ketyl is more strongly bound to acetone than the dimethyl ketyl to the corresponding aromatic ketone. 
The enthalpy barrier for $\mathrm{H}$ atom transfer with respect to the complex varies significantly from $28.1 \mathrm{~kJ} \mathrm{~mol}^{-1}$ of Bp with substitution of the aromatic rings. Methoxy substitution results in the increase of the barrier height, $\mathrm{CF}_{3}$ substitution decreases it. The tendency is in agreement with that of the reaction enthalpies, but the free energy relationship is not linear.

\section{DISCUSSION}

\section{Substituent effect on the photophysical properties of benzophenone derivatives}

All four derivatives we studied have qualitatively similar absorption spectra in $n$-hexane: there is a well resolved symmetry forbidden $n-\pi *$ transition in the near UV spectral region followed by more intense bands peaking around $250 \mathrm{~nm}$ corresponding to the allowed $\pi$ - $\pi^{*}$ transitions (Figure 1). The methoxy derivative deviates from the other studied ketones: the oscillator strength of the $n-\pi^{*}$ band is slightly larger than that for the other three compounds, and at the same time the higher energy intense peak is shifted to the red (see Figure S1 and Table S1). The increase of solvent polarity results in the expected effect: the $n-\pi^{*}$ state has a moderate hypsochromic, the $\pi-\pi^{*}$ state a bathochromic shift (or nothing as can be see in Figure $1 \mathrm{~S}$ for CFBp). In the case of MeOBp the energy difference between the $n-\pi^{*}$ and $\pi$ - $\pi^{*}$ bands decreases so much in $\mathrm{MeCN}$ that the maximum of the absorption band of the lowest excited state can not be separated. Furthermore, the $n-\pi^{*}$ transition gains further intensity from the upper states resulting in an additional increase of the corresponding transition dipole moment (see Table 1 and Table S1).

In luminescence spectroscopy, the usual way to determine the thermodynamic singlet-singlet and singlet-triplet energy gap is that the respective absorption and emission spectra normalized to the maximum (more properly to the maximum of the $0-0$ bands) are plotted together and the crossing 
point of the two curves is taken to be the ground-to-first excited singlet or triplet energy difference (data listed in tabulations are also determined using this procedure). However, for benzophenone derivatives this method can not be used because, concerning the singlet-singlet gap, the fluorescence, and for the singlet-triplet gap, the triplet $\leftarrow$ ground state absorption spectra are not available. Kearns and $\mathrm{Case}^{31}$ measured the phosphorescence excitation spectra for Bp and MeOBp in EET glass with so high sensitivity that the vibronic structure of the triplet $\leftarrow$ singlet absorption was also resolved. A 570 and $330 \mathrm{~cm}^{-1}$ 0-0 peak separation was observed for Bp and MeOBp, respectively. Adopting their data we increase by $3 \mathrm{~kJ} \mathrm{~mol}^{-1}$ (approx. half of the Stokes shift) the 0-0 Franck-Condon triplet energy of phosphorescence in polar solvents and by $2 \mathrm{~kJ}$ $\mathrm{mol}^{-1}$ in alkanes to get the data appropriate for further compilation (see Table 1). In a similar way, the FC 0-0 data of singlet absorption were decreased by the same amount to derive singlet energies comparable to the thermodynamic values reported in compilations (see Table 1). These corrected triplet data show good agreement (considering the unavoidable uncertainties increased by the correction mentioned before) with triplet energies given for $\mathrm{Bp}$ and $\mathrm{MeOBp}$ in ethyl acetate in the recent comprehensive compilation of Merkel and Dinnocenzo ${ }^{21 \mathrm{~b}}$.

The change of the triplet emission spectra and the singlet-triplet energy gap derived as described above with variation of the substituent and the polarity of the solvent is similar to that observed for the $n-\pi^{*}$ absorption, as expected. It is worth noticing, however, that in EPA glass matrix at $-192{ }^{\circ} \mathrm{C}$ one can observe an additional blue shift compared with butyronitrile, as large as $200-$ $600 \mathrm{~cm}^{-1}$ (see Table 2) as if EPA glass represented an extremely polar environment. This is, however, not so: the reason for the hypsochromic effect is that in EPA glass not the ketone itself but its hydrogen-bonded complex absorbs light. The ketones form hydrogen bonded complexes 
with the ethanol molecules of the frozen solvent. Under our experimental conditions most of the hydrogen bond acceptor molecules are complexed ${ }^{20}$ for two reasons: the alcohol concentration is high, and at the very low temperature the equilibrium constant of complexation is relatively large. The hydrogen-bond complexation induces a strong blue shift, larger than the most polar solvent we studied. This indicates a caveat is in place concerning the reliability of triplet energies derived from measurements made in alcohol-containing glasses such as EPA or EET (ether / ethanol / toluene).

The location of the $n-\pi^{*}$ absorption maximum as well as the singlet energy increases monotonously in the sequence of dpCFBp, CFBp, Bp and MeOBp, and the same applies to the triplet energy (Table 1). This order does not completely match that of the Hammett parameters, dpCFBp and CFBp being interchanged. Hammett's relations were applied successfully several times $^{32,33}$ to account for the substituent effect on the fluorescence behavior. Moreover, Leight and coworkers $^{34}$ studied the dependence of reduction potential and triplet energy of benzophenone derivatives on the chemical nature of ring-substituents, and found good Hammett correlations for half-wave reduction potentials. ${ }^{34 b}$ They explained the tendency in what they call merostabilization by the change of Hammett parameters both for reduction potential and triplet energy. However, inspection of the correlation of the triplet energy with the Hammett parameters shown in their paper indicates it is rather poor. Examining our data one can conclude that the para-substituted benzophenones more or less follow the expected tendency; however the bismeta substituted CFBp is definitely out of the predicted order. From the Hammett relationship one can expect a much larger blue shift for CFBp than for dpCFBp (the $\Sigma \sigma$ parameters are $1.66^{35 a}$ or $1.72^{35 \mathrm{~b}}$ and $1.10^{35 \mathrm{a}}$ or $1.07^{35 \mathrm{~b}}$, respectively) contrary what was observed experimentally. A 
possible explanation could be that the effect of several substituents is not perfectly additive. However, as it will be shown below, the additivity assumption works quite well in other contexts. This suggests that the behavior of the energetics of the $n-\pi^{*}$ singlet state is determined by factors other than those accounted for by the Hammett constants.

The aromatic ketones undergo hydrogen bonded complex formation in the presence of aliphatic alcohols. The equilibrium constant increases with the electron donating strength of the substituent (which enhances the electron density on the oxygen atom) from CFBp to MeOBp as it is shown for the case of a good hydrogen bond donor, HFIP in $n$-hexane (Table 1). The complex formation causes an approx. $1000 \mathrm{~cm}^{-1}$ hypsochromic shift of the $\mathrm{n}-\pi^{*}$ absorption band of all four compounds. This means that the equilibrium constants of complex formation are approx. 160 times smaller in the singlet excited state than in the ground state, inducing a driving force for dissociation of the excited ketone - HFIP complex. ${ }^{20}$ Using isopropyl alcohol as hydrogen bond donor in $\mathrm{MeCN}$, the corresponding equilibrium constants must be at least two orders of magnitude lower than those for HFIP in $n$-hexane, although qualitatively the effect probably remains similar. The reduction of the equilibrium constant of complex formation is manifested in a slight but well recognizable blue shift of the absorption maximum by complexation (1.2 nm, corresponding to approx. $30 \%$ decrease of the equilibrium constant in the singlet state compared to the ground state).

\section{The ketyl radical - ketyl radical reactions}

The effect of the substituents on the rate parameters of second order radical - radical reactions (3), (4) and (6) (see Table 3) at first sight is quite surprising. From the previous studies of the triplet unsubstituted benzophenone - 2-propanol - acetonitrile system the following qualitative 
picture has arisen: the aromatic ketyl self-reaction (6) is an activation controlled combination whose rate constant at room temperature is an order of magnitude lower than that of the aliphatic ketyl self- (3) and cross-reaction (4), both being dominated by the disproportionation process. For both trifluoromethyl-substituted aromatic ketyl radicals, reaction (6) proved to be faster by an order of magnitude than the reference self combination of the unsubstituted diphenyl ketyl radical (see Table 3), despite the fact that both of these radicals are the most stable (see Table S3 and Figure 9). One may find a reasonable explanation for this contradiction by considering that the ketyl radicals of the fluorinated derivatives form hydrogen-bonded complexes less readily. The consequence is that most of them are free in the acetonitrile - isopropyl alcohol solvent mixture, contrary to the unsubstituted benzophenone-ketyl radicals. The uncomplexed radicals are expected to be more reactive than the complexed form, because in the latter case the radical center is shielded by the hydrogen bond donor. This hypothesis is supported by our earlier observation $^{16 a}$ that the rate coefficient of the combination reaction (6) of the aromatic ketyl radical derived from benzophenone increases considerably with the decrease of the alcohol concentration when the latter is low (see Figure 2 in reference 16a). This effect is especially well expressed when the hydrogen donor is benzhydrol which has a larger steric hindrance (and shielding effect) and forms a more stable complex. Moreover, from the measurements made in the benzophenone - benzhydrol system one can conclude that in the case of the tetratrifluoromethyl substituted derivative new combination channels (such as LAT formation) open in the aromatic ketyl self-reaction process, which surely increases the overall rate constant $k_{6}$.

The rate coefficient of the cross-combination reactions is expected to be twice as large as the geometric mean of the rate constants of the two related self-termination processes. ${ }^{36}$ For the cross-reaction (4) this relation applies only in case of MeOBp. For the other derivatives $k_{4}$ is 
subsequently larger than twice the geometric mean of $k_{3}$ and $k_{6}$, and the deviation increases with increasing electron withdrawing strength of the ring substituents. This is not surprising, considering that reaction (4), contrary to the radical self termination processes, has more channels, such as disproportionation or the efficient LAT forming reactions, whose rate certainly depends on the nature and position of the ring substituents.

\section{The hydrogen atom transfer reaction (5)}

The hydrogen atom metathesis reaction is characterized by a preexponential factor of around $10^{8}$ $\mathrm{dm}^{3} \mathrm{~mol}^{-1} \mathrm{~s}^{-1}$ which is typical for this type of reaction. ${ }^{12}$ Naguib and coworkers ${ }^{14}$ proposed a "spatially resolved electron - proton transfer" mechanism for this kind of reaction, involving a polar transition state. There are several facts that undermine such a mechanism.

1) The magnitude of the measured preexponential factor does not support such a model (note that the derivation of the preexponential factor in Ref. 14 proved to be incorrect ${ }^{13}$ ).

2) Even stronger evidence against the participation of a polar transition state is the effect of solvent polarity on the rate constant of reaction (5). A dipolar transition state corresponding to simultaneous electron - proton transfer would be stabilized more efficiently by polar solvents, so that the rate should increase with increasing solvent polarity. According to our previous experiments, ${ }^{13}$ (as well as shown in the present work) however, $k_{5}$ considerably decreases with increasing solvent polarity, discrediting the hypothesis of a polar transition state.

3) According to our electronic structure calculations, the transition state is very similar to those of other $\mathrm{H}$-atom transfer reactions between two radicals, it shows no sign of simultaneous electron - proton transfer and its charge distribution is very similar to those of the reactant $\mathrm{Bp}$ derivatives. Thus, one can not expect extra solvent stabilization of the TS with respect to the 
reactants. Inspecting the transition structures and the van der Waals complexes formed by aliphatic ketyl radicals and $\mathrm{Bp}$ or its derivatives, one can see that it is hard to imagine how electron transfer could take place. The possible target of an electron to be transferred can be one of the aromatic rings (in addition to the carbonyl oxygen, but then the proton and electron are not transferred separately but together as a $\mathrm{H}$ atom). For an electron-transfer pathway separate from that of the proton, there should be a bridge when the two reactants are close. The aliphatic ketyl radical, in addition to the $\mathrm{OH}$ group participating in the reaction, has only methyl groups, which in principle could serve as an electron-transfer bridge, but it is hard to find a driving force for an electron to hop from a spatially separated $\mathrm{CH}_{3}$ group to an aromatic ring.

As mentioned above, the quantum chemical calculations yielded transition state structures for reaction (5ii) typical to $\mathrm{H}$ atom abstraction reactions. In the reactions of substituted benzophenones with the aliphatic ketyl, the hydrogen atom is closer to the oxygen of the aliphatic ketyl radical than to that of the aromatic ketone, i.e. the reactions are characterized by an early barrier as expected for exothermic reactions. In agreement with Hammond's principle, the barrier height for reaction (5) decreases as the exothermicity increases (see the inset in Figure $10)$.

The electronic structure calculations also show that the secondary hydrogen atom transfer (5) involves formation of a hydrogen bonded complex. Thus the rate coefficient and Arrhenius parameters of reaction (5) we measured are "virtual" data, because they correspond to a combination of several elementary steps. Further complicates the kinetics that both species involved in the reaction, in particular, the aliphatic ketyl radical can also form complexes with IPA and $\mathrm{CH}_{3} \mathrm{CN}$, both present in large concentration. Based on the information from the 
quantum chemistry calculations, in addition to those listed in Scheme 1, reactions shown in Scheme 3 are also possible in the $\mathrm{Bp}$ - aliphatic ketyl radical - 2-propanol -acetonitrile system, and can have a role in determining the measurable value of $k 5$. 


\section{Scheme 3.}

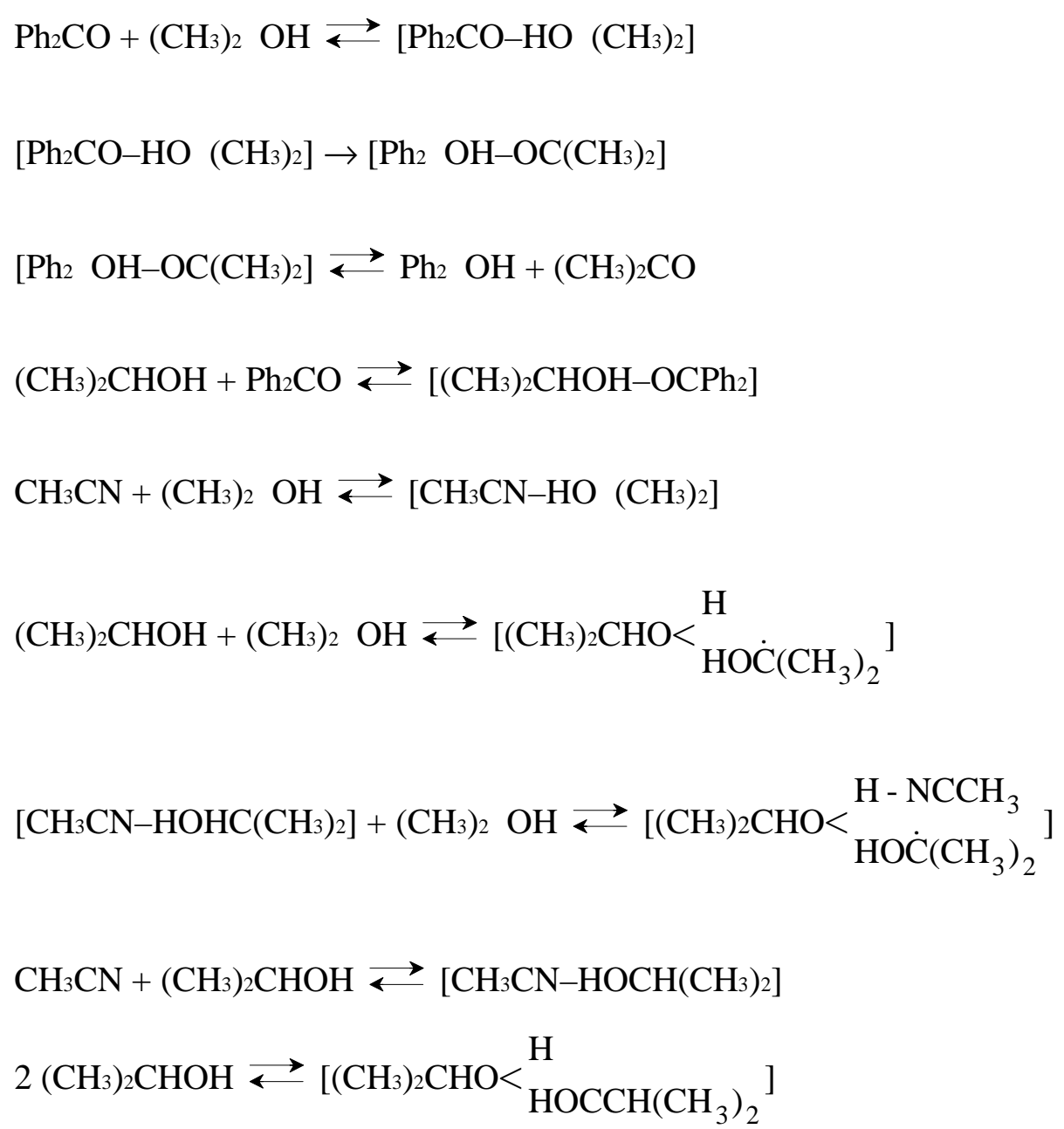

Let's first consider the equilibrium determining the concentration of the Bp reactant, the complexation of Bp by the alcohol, reaction (7). We measured its formal equilibrium constant in acetonitrile solution and found $K_{7}(\mathrm{MeCN})=0.038 \pm 0.005 \mathrm{dm}^{3} \mathrm{~mol}^{-1}$. This low value means that the ground-state ketone is essentially in the uncomplexed form in the IPA $-\mathrm{CH}_{3} \mathrm{CN}$ solvent mixture, so that the influence of reaction (7) on the kinetics may be disregarded. (The formal $K_{7}$ value is low because the concentration of benzophenone is four orders of magnitude lower than 
that of acetonitrile, and as a consequence most of the 2-propanol molecules are complexed not to benzophenone but to the solvent according to reaction (9) and (10).)

The other reactant, the aliphatic ketyl can also form hydrogen-bonded complexes not only with BP but also with both constituents of the 2-propanol - acetonitrile solvent mixture. Our measurements of the rate coefficient of reaction (5) in acetonitrile with varying amounts of IPA are instructive in this respect: the measured rate coefficient, $k_{5}$ is relatively large at low IPA concentration, and decreases to a limiting value at higher [IPA]. This can be explained by considering that the aliphatic ketyl radical, if it donates the $\mathrm{H}$ atom of its $\mathrm{OH}$ group to form a hydrogen bond with IPA or MeCN, is not apt for reacting with Bp. Then, the rate is determined by the concentration of the ketyl-Bp complex, which is in equilibrium with the benzophenone and free aliphatic ketyl radical.

$$
R_{5}=-\frac{d[\mathrm{Bp}]}{d t}=k_{5 i i} K_{5 i}\left[\left(\mathrm{CH}_{3}\right)_{2} \dot{\mathrm{COH}}\right]^{\text {free }}[\mathrm{Bp}]
$$

where $[\mathrm{Bp}]$ and $\left[\left(\mathrm{CH}_{3}\right)_{2} \dot{\mathrm{COH}}\right]^{\text {free }}$ stand for the concentration of the (bare or substituted) Bp and the free aliphatic ketyl radical. The concentration of the latter is what is left from the nominal ketyl radical concentration reduced by the fractions bound in the complexes with MeCN, IPA, [MeCN-IPA] and Bp. Taking into account the simultaneous equilibria (8i), (8ii) and (5i) influencing the concentration of the aliphatic ketyl radical, one gets

$$
R_{5}=k_{5 i i} \cdot \frac{K_{5 i}[\mathrm{Bp}]}{1+K_{8 i}[\mathrm{MeCN}]+K_{8 i i}[\mathrm{IPA}]+K_{9} K_{8 i i i}[\mathrm{IPA}][\mathrm{MeCN}]+K_{5 i}[\mathrm{Bp}]}\left[\left(\mathrm{CH}_{3}\right)_{2} \dot{\mathrm{COH}}\right]
$$

and the measurable rate coefficient is 
$k_{5}=\frac{k_{5 i i} K_{5 i}}{1+K_{8 i}[\mathrm{MeCN}]+K_{8 i i}[\mathrm{IPA}]+K_{9} K_{8 i i i}[\mathrm{IPA}][\mathrm{MeCN}]+K_{5 i}[\mathrm{Bp}]}$.

The last term in the denominator is negligible because $[\mathrm{Bp}]$ is small, so the net order of the reaction in fact is two. In the limit of small alcohol concentration, the measurable rate coefficient is

$k_{5}^{0}=\frac{k_{5 i i} K_{5 i}}{1+K_{8 i}[\mathrm{MeCN}]}$,

and from this value it decreases when [IPA] increases. The other limiting case is when [IPA] is very large. Formally, in that limit formula (13) would yield zero for the rate coefficient. This limit, however, can not be achieved, because the IPA concentration is limited by its value in neat IPA. In fact, the model for $k_{5}$ could include the complexation of IPA by MeCN and by IPA (reactions (9) and (10)), and consider $[\mathrm{MeCN}]$ so large that it would not change due to complexation. That model yields a finite virtual rate coefficient in the limit of high alcohol concentration, but the low-[IPA] limit would be essentially the same as in (14). The conclusion is that the exact shape of the $k_{5}$ curve as a function of [IPA] is described by a relatively complicated equation, but the accuracy of the measurements does not warrant an attempt to derive the rate coefficients and equilibrium constants by fitting the formula to experimental data. Equations (13) and (14) indicate that the rate coefficient is a complex function involving several rate coefficients and equilibrium constants (even in the small [IPA] limit), each having its own temperature dependence. Accordingly, the activation energy measured for $k_{5}$ will not be directly connected to the activation enthalpy calculable from the parameters of the potential barrier corresponding to the transition structure of reaction (5). The quantum chemistry calculation on the energetics of complex formation allows us to conjecture about the magnitude of the difference between the 
calculated activation enthalpy and the measured activation energy. The calculated reaction enthalpy of complex formation in reactions (8i), (8ii) and (8iii) is $-13,-21.6$ and $-26.9 \mathrm{~kJ} \mathrm{~mol}^{-1}$, respectively (Table S3). This means that the equilibrium constant for complexation of the aliphatic ketyl with 2-propanol is more than a magnitude larger than that with the solvent acetonitrile. At the concentration of the rate coefficient measurement, [IPA] $=1.31 \mathrm{~mol} \mathrm{dm}^{-3}$, the ketyl radical tends to be complexed more by the alcohol and the alcohol-acetonitrile complex than by acetonitrile (whose concentration is about $18 \mathrm{~mol} \mathrm{dm}^{-3}$ ). To simplify the picture, we assume that there is a single term in the denominator of Eq. (13) that represents all three complexes, and that the temperature dependence of this term is determined by, as a representative value, the reaction enthalpy of reaction (8ii). At a given solvent composition the denominator of Eq. (13) is the same for all benzophenone derivatives, so that the contribution of complex formation to the activation energy of reaction (5) is independent of the substitution of benzophenone. Figure 10 shows the correlation between the calculated activation enthalpy $\Delta H_{5}^{\#}$, shifted by $-\Delta H_{8}=21.6 \mathrm{~kJ} \mathrm{~mol}^{-1}$ and the measured activation energy $E_{5}$ with the overall Hammett parameter of the substituent. The applied shift happens to yield very good agreement between $\Delta H_{5}{ }^{\#}$ and $E_{5}$, indicating that the suggested picture is not far from reality. The Hammett parameter proves to be a good measure of the substituent effect in this case: the activation parameters show a good linear correlation with it.

\section{CONCLUSIONS}

The measurements and calculations of the spectroscopic, photophysical and photochemical properties of the substituted benzophenones yielded clear tendencies and monotonous changes. The reaction and activation energies correlate well with the overall Hammett parameter of the 
substituents of the aromatic ring. The photoreduction reactions (2) and (5) are characterized by linear free energy relationships: the measured activation energy as well as the calculated barrier height and location correlate very well with the reaction enthalpy. The driving force, the enthalpy of the reaction depends essentially on the stabilization energy of the ketyl radical compared with the ketone it is derived from. The trifluoromethyl substituted ketyl radicals are much more stable than those containing electron donor substituents and accordingly their photochemical reactions are faster. Concerning electron-donor substituents, our studies show that, contrary to what is suggested in the literature. ${ }^{12}$ even MeOBp behaves and reacts as an $n-\pi^{*}$ excited ketone ${ }^{28}$ (which is supported by the properties of its phosphorescence spectrum), and the smaller reaction rate in its photoreduction steps is caused by their lower reaction exothermicity.

The experimentally measurable activation energy, especially that of the secondary photoreduction step (5) can not be assigned to any single elementary step, because in the polar acetonitrile solvent, containing a large amount of the hydrogen-bond donor alcohol reactant, multiple complex formation equilibria are established that mask the activation energy of the actual hydrogen atom transfer step. 
FIGURES:

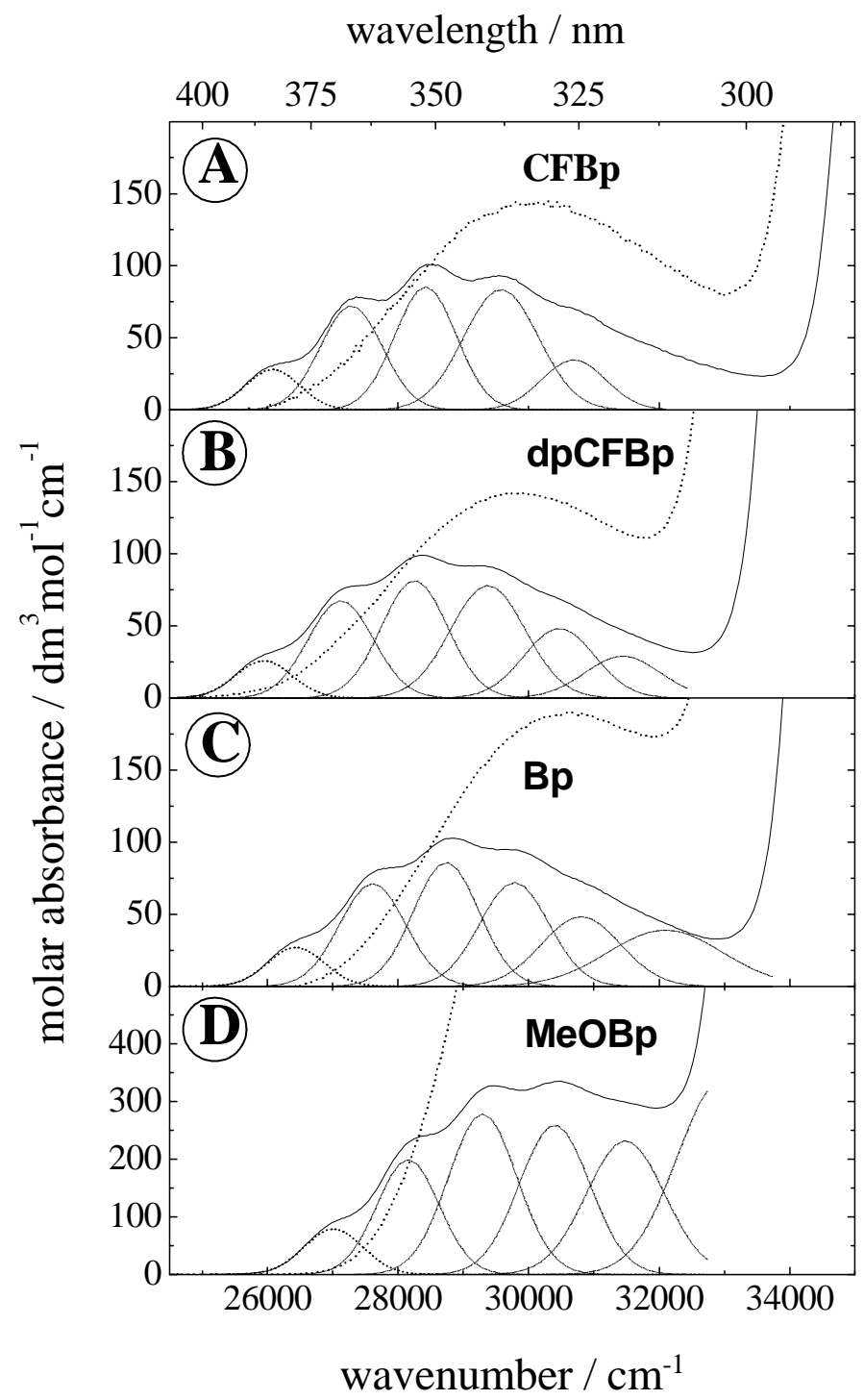

Figure 1. Absorption spectra of the lowest transition of CFBp (A), dpCFBp (B), Bp (C) and MeOBp (D) in $n$-hexane at room temperature. An approximate fit of the vibronic bands (dotted line), as well as the spectra of the 1:1 complexes of the Bp derivatives with HFIP (dashed line) are also indicated. 


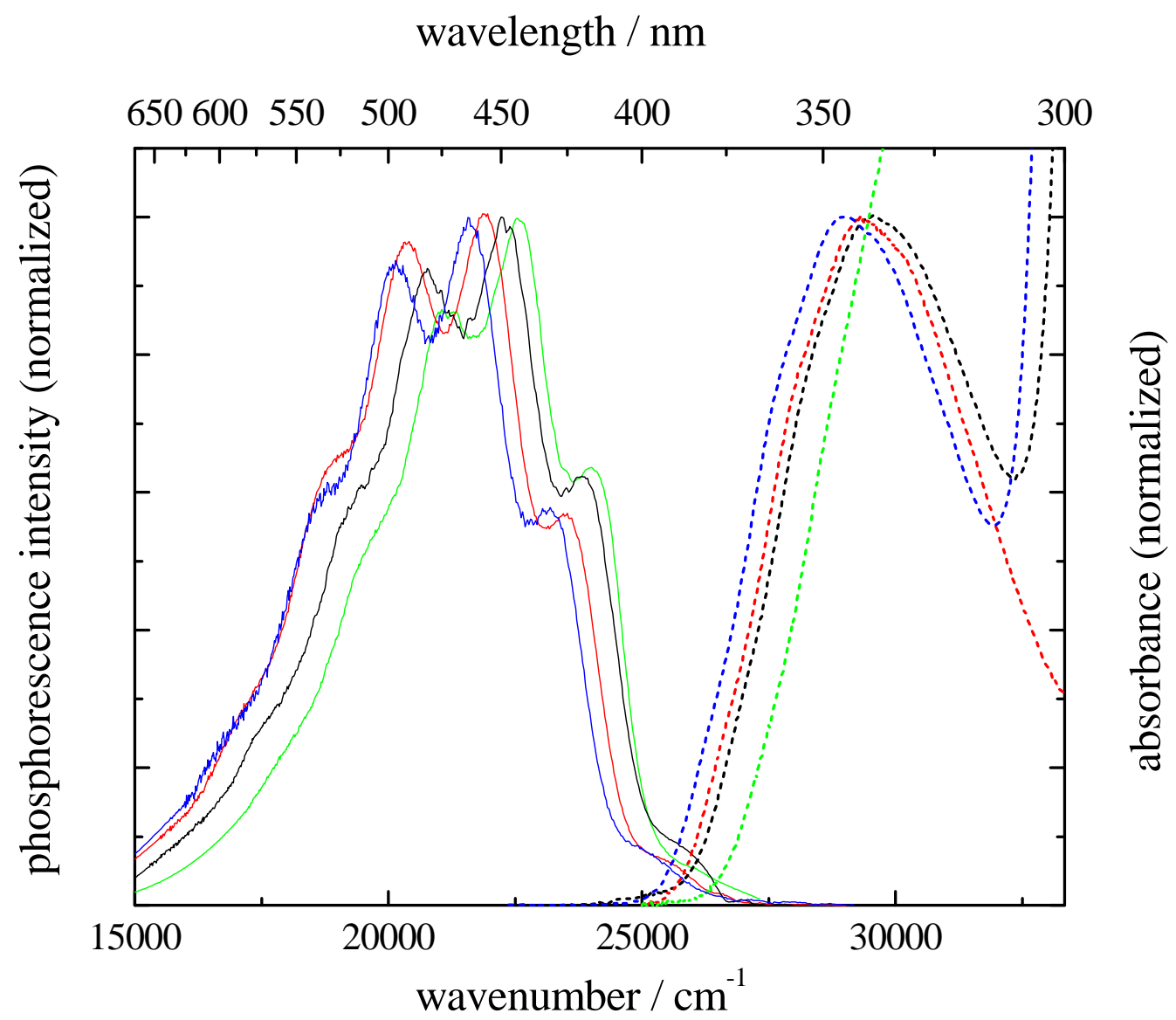

Figure 2. Phosphorescence spectra of CFBp (red), dpCFBp (blue), Bp (black) and MeOBp (green) in $\mathrm{MeCN}$ at $25{ }^{\circ} \mathrm{C}$ together with the corresponding absorption spectra (dashed line). 


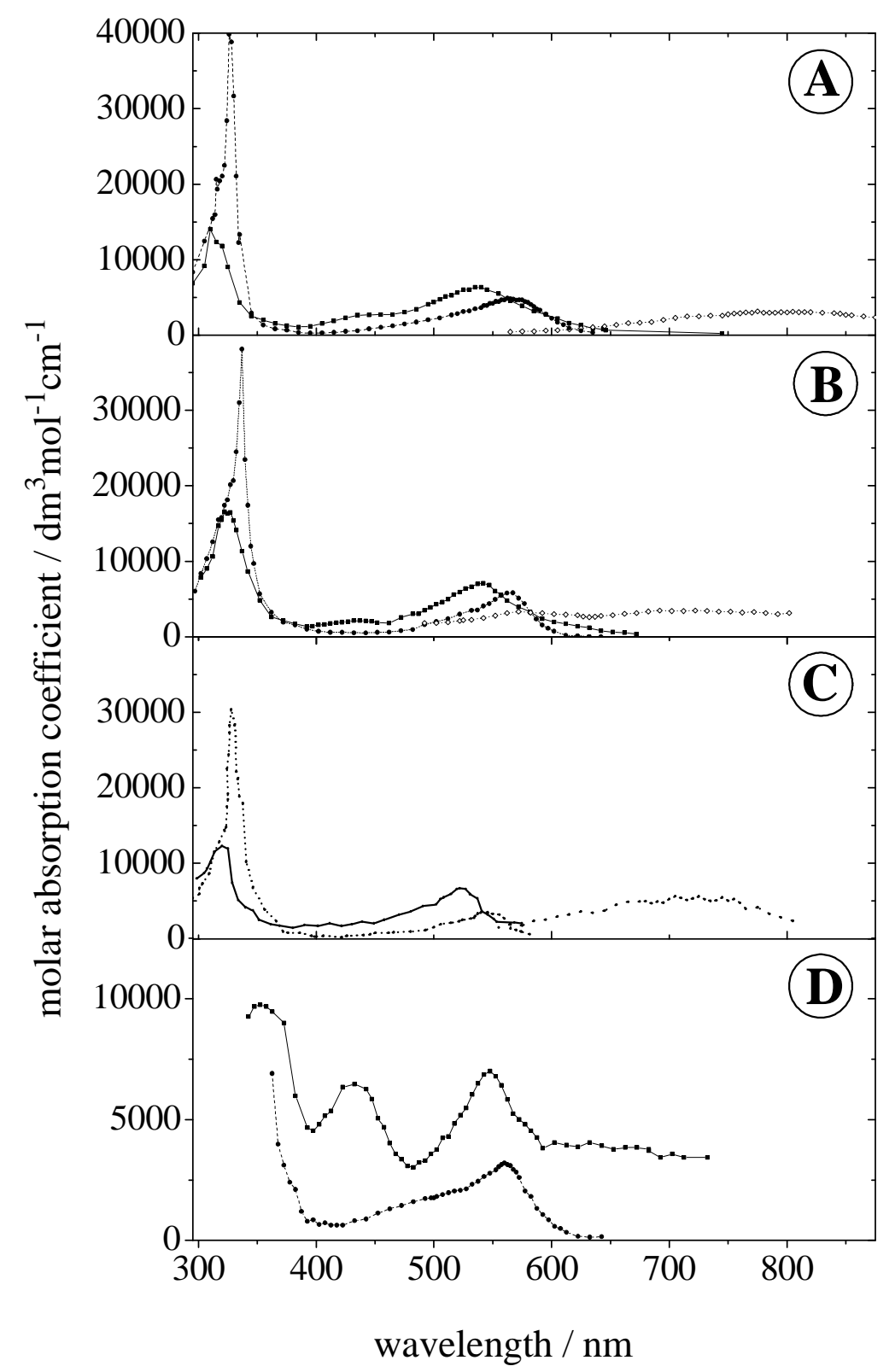

Figure 3. Transient absorption spectra of the triplet ketone (square, full line) and the radical anion (empty diamond, dotted line) in acetonitrile, as well as of the ketyl radicals (circle, dashed line) in $1.31 \mathrm{~mol} \mathrm{dm}^{-3} \mathrm{IPA}$ in acetonitrile for CFBp (A), dpCFBp (B), Bp (C) and MeOBp (D, in neat IPA). 


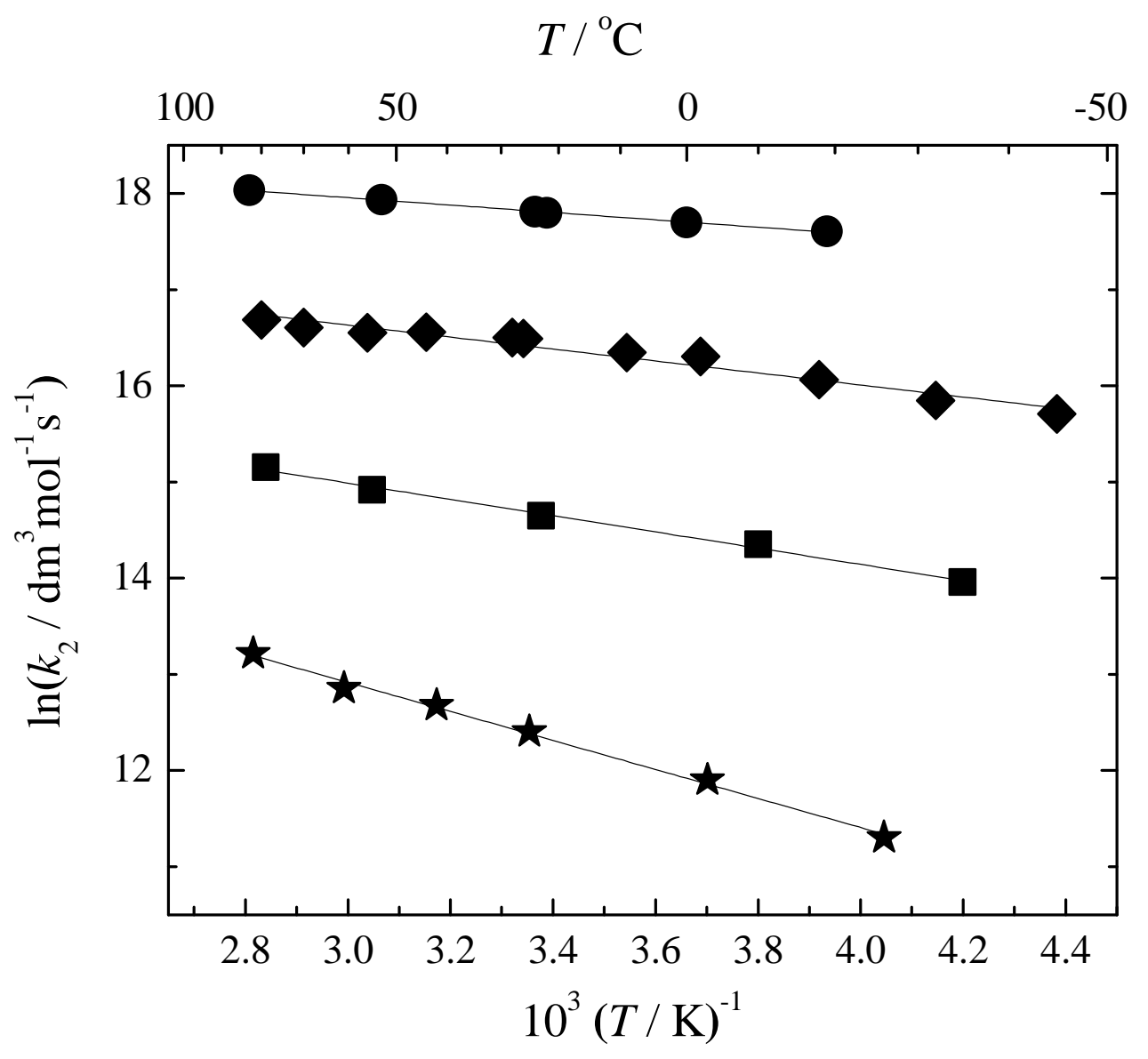

Figure 4. Arrhenius plot of the reaction rate coefficient of the primary photoreduction reaction (2) of benzophenone derivatives (CFBp: circle. dpCFBp: diamond, Bp: square, MeOBp: star) by propan-2-ol in acetonitrile. 


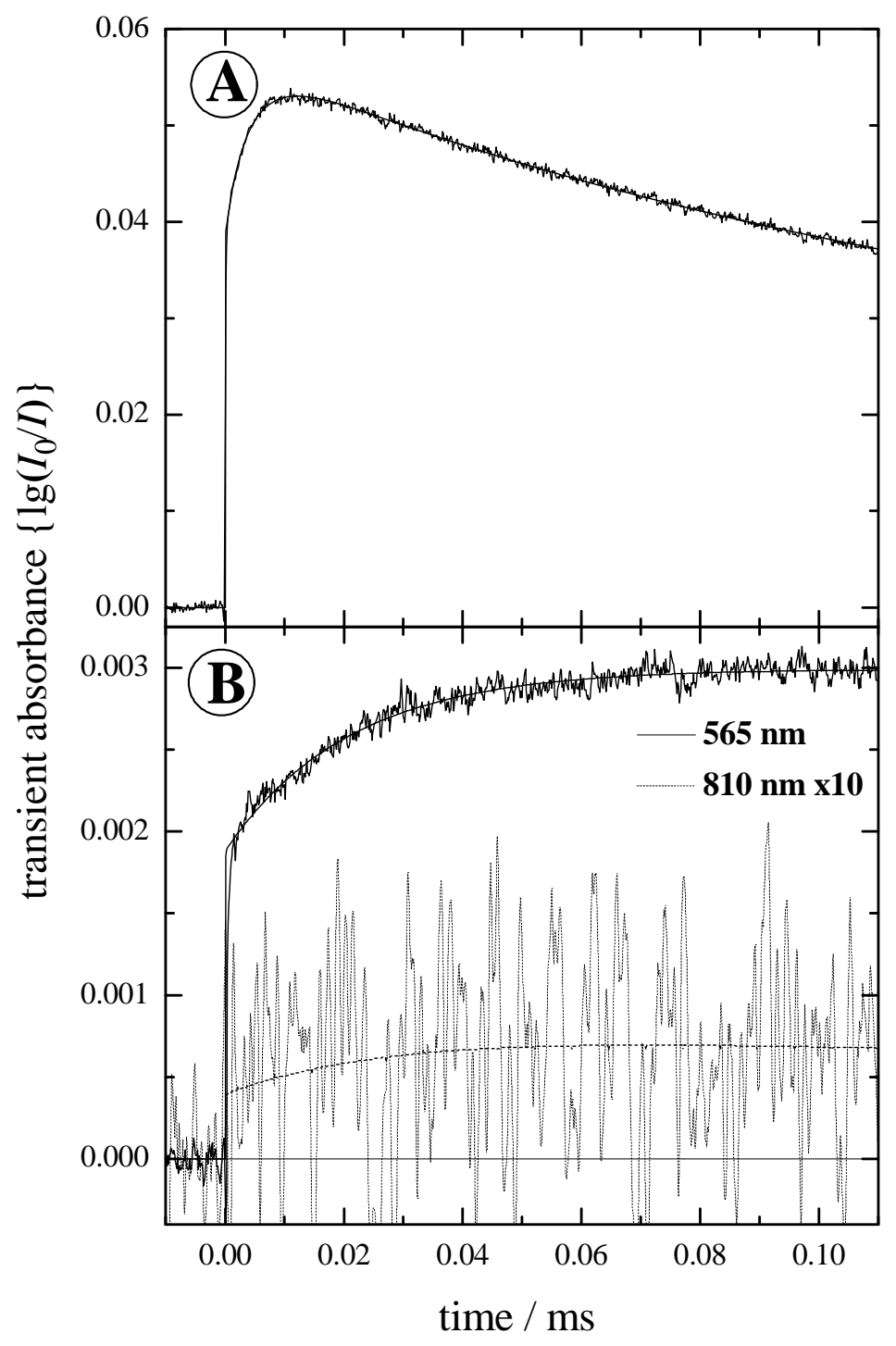

Figure 5. Time dependent absorbance of CFBp in $1.31 \mathrm{~mol} \mathrm{dm}^{3} \mathrm{IPA}-\mathrm{MeCN}$ solvent at room temperature $\left(23{ }^{\circ} \mathrm{C}\right.$ ) applying (A) high (approx. $\left.50 \mathrm{~mJ} / \mathrm{flash},[\mathrm{CFBp}]=0.008 \mathrm{~mol} \mathrm{dm}^{-3}\right)$ and (B) low energy laser excitation (approx. $8 \mathrm{~mJ} / \mathrm{flash}[\mathrm{CFBp}]=0.0012 \mathrm{~mol} \mathrm{dm}^{-3}$ ). Excitation: at 308 nm, detection: at 565 (black) and 810 (dashed line, multiplied by 10) $\mathrm{nm}$. 


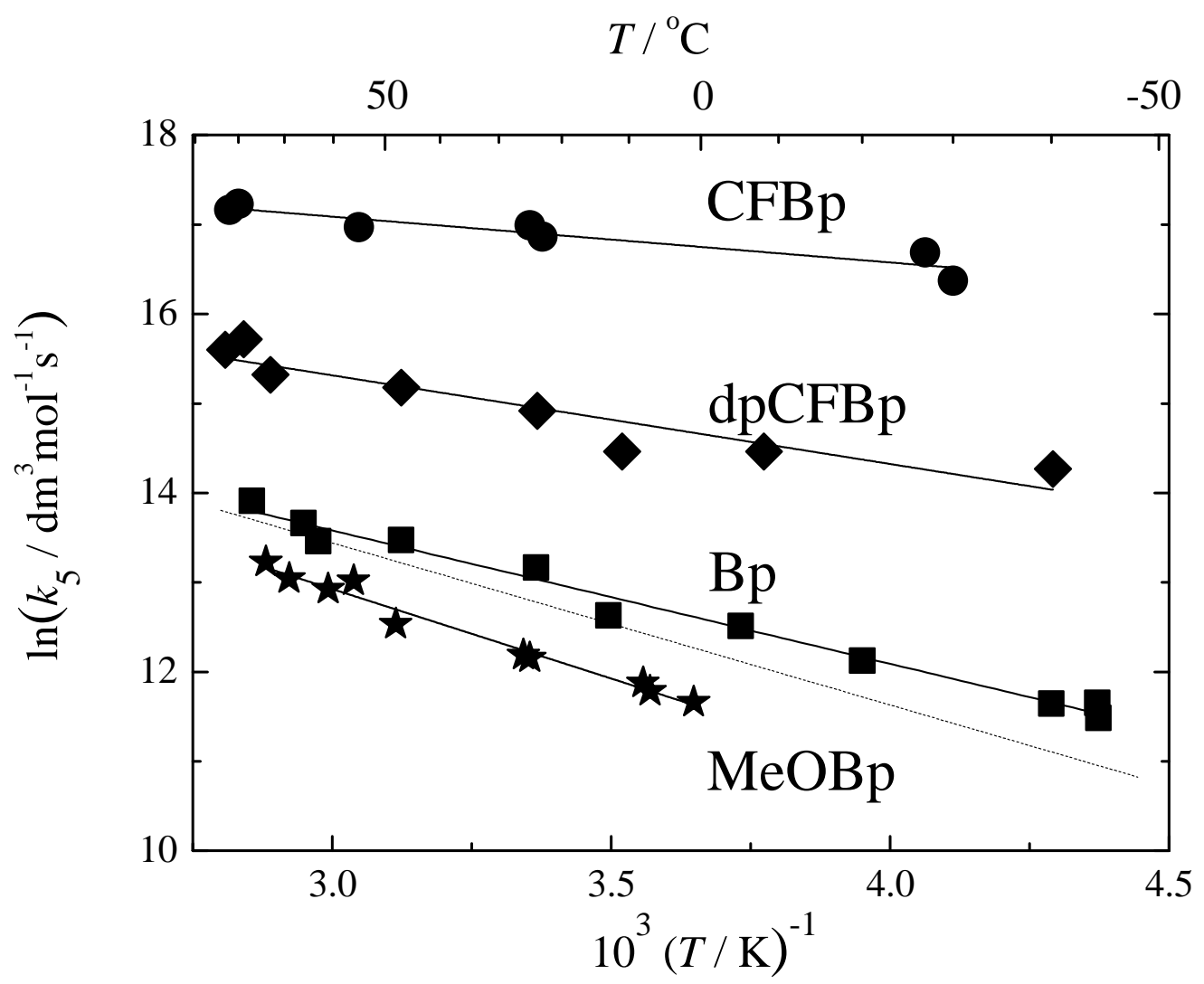

Figure 6. Arrhenius plot of the reaction rate coefficient of the secondary photoreduction reaction (5) of benzophenone derivatives (CFBp: circle. dpCFBp: diamond, Bp: square, MeOBp: star) in $1.31 \mathrm{~mol} \mathrm{dm}^{-3}$ propan-2-ol - acetonitrile solvent. (The dashed line is derived from the published ${ }^{13}$ data measured for Bp in $2.62 \mathrm{~mol} \mathrm{dm}{ }^{-3}$ propan-2-ol - acetonitrile solvent mixture.) 


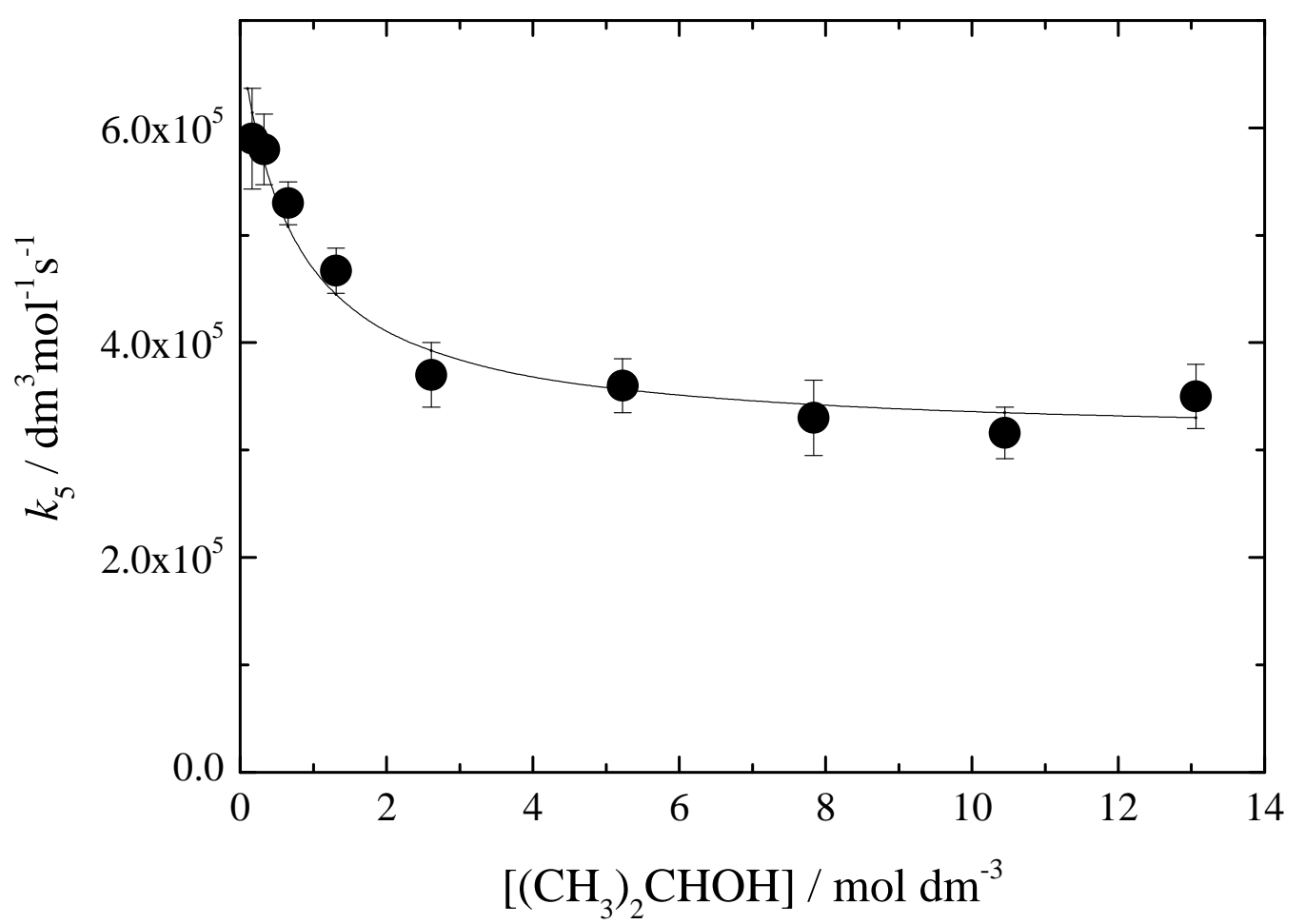

Figure 7. The reaction rate coefficient (with $1 \sigma$ uncertainty) of the secondary photoreduction reaction (5) of unsubstituted benzophenone by IPA as the function of the alcohol concentration in acetonitrile at $25^{\circ} \mathrm{C}$. 


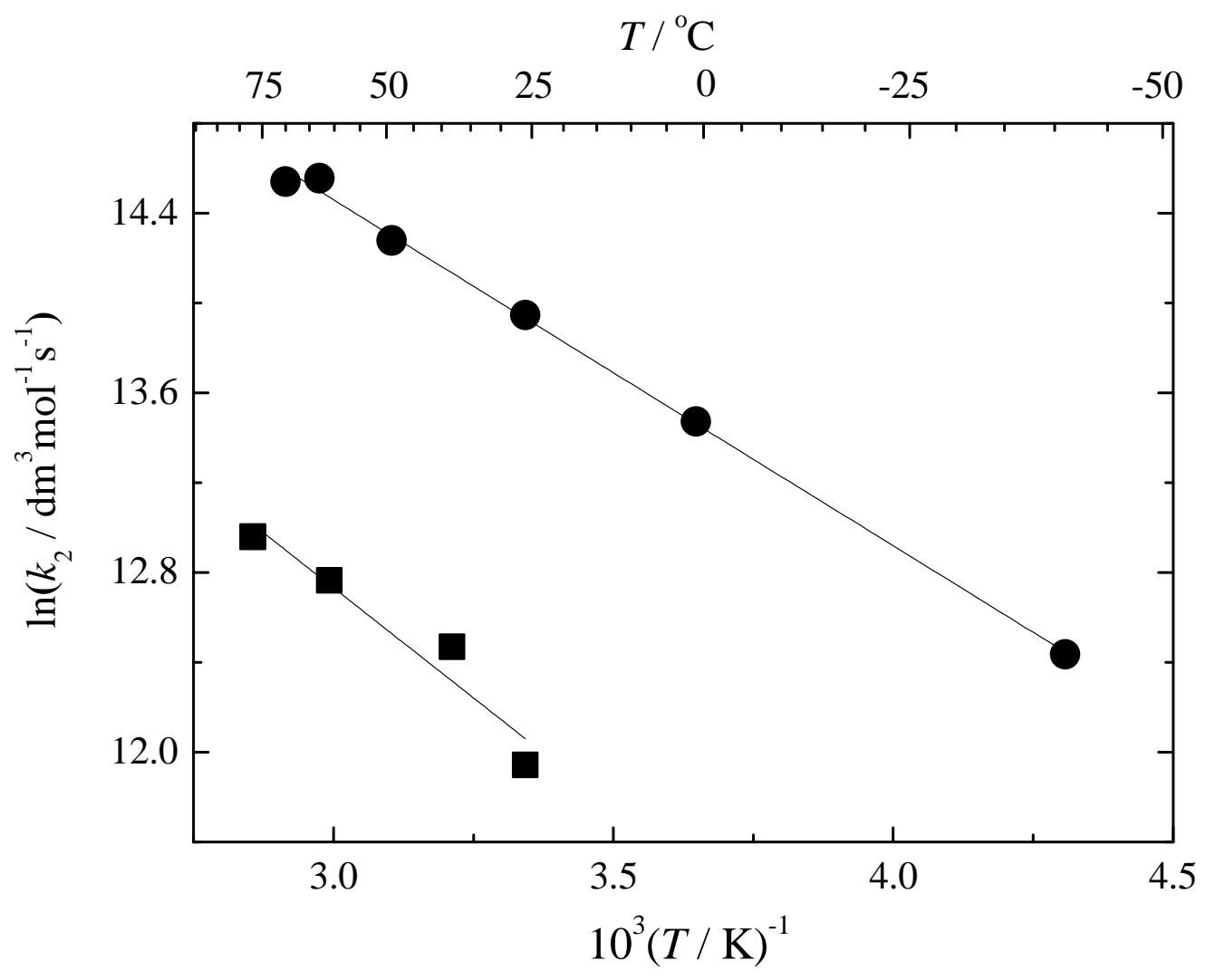

Figure 8. Arrhenius plots of the reaction rate coefficient of the primary photoreduction reaction (2) of triplet CFBp (circle) and Bp (square) with GVL in acetonitrile. 


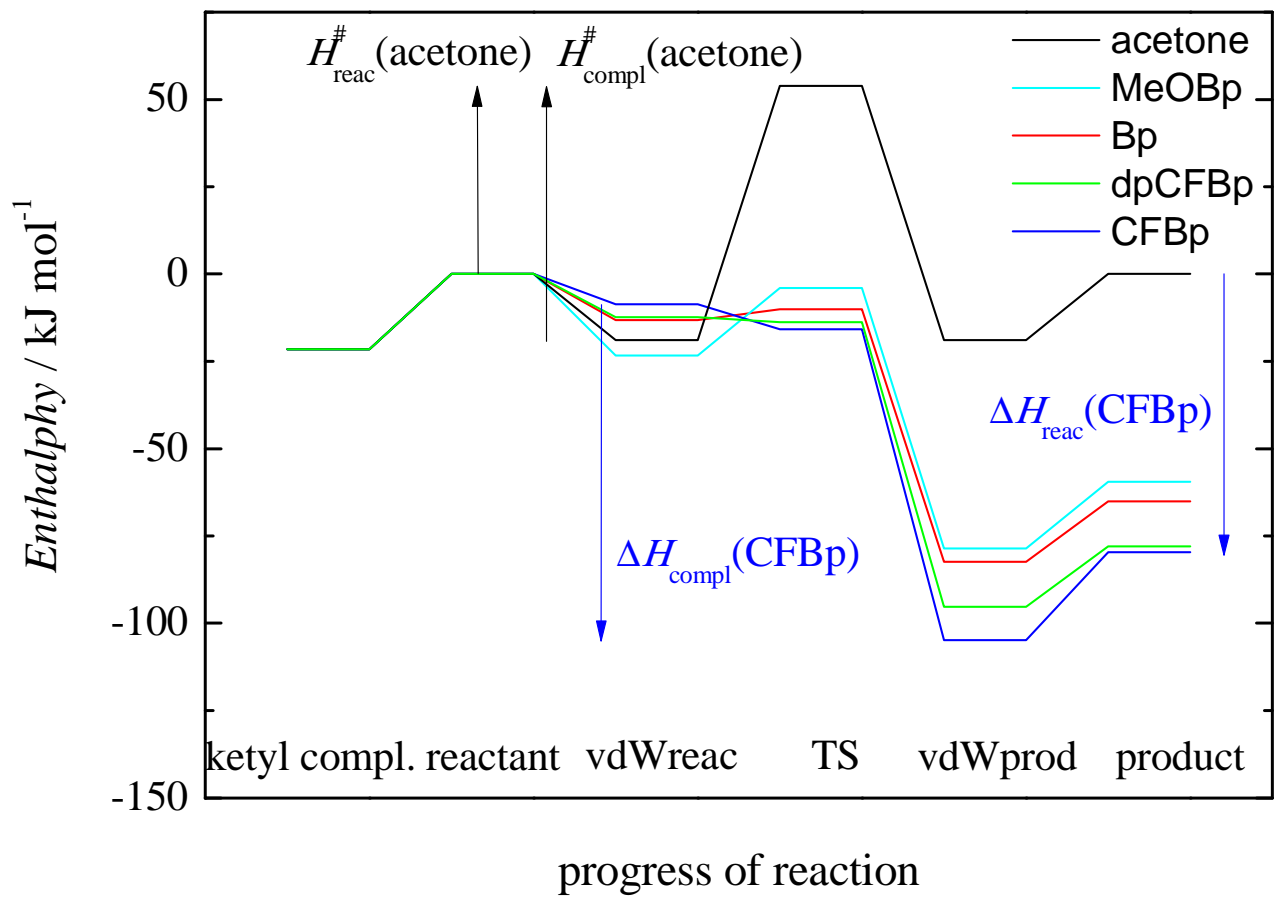

Figure 9. The calculated enthalpy profile of the ketone-ketyl radical $\mathrm{H}$ atom transfer reactions in acetonitrile. The meaning the corresponding reaction enthalpies for CFBp, the TS energies for acetone is indicated on the figure. 


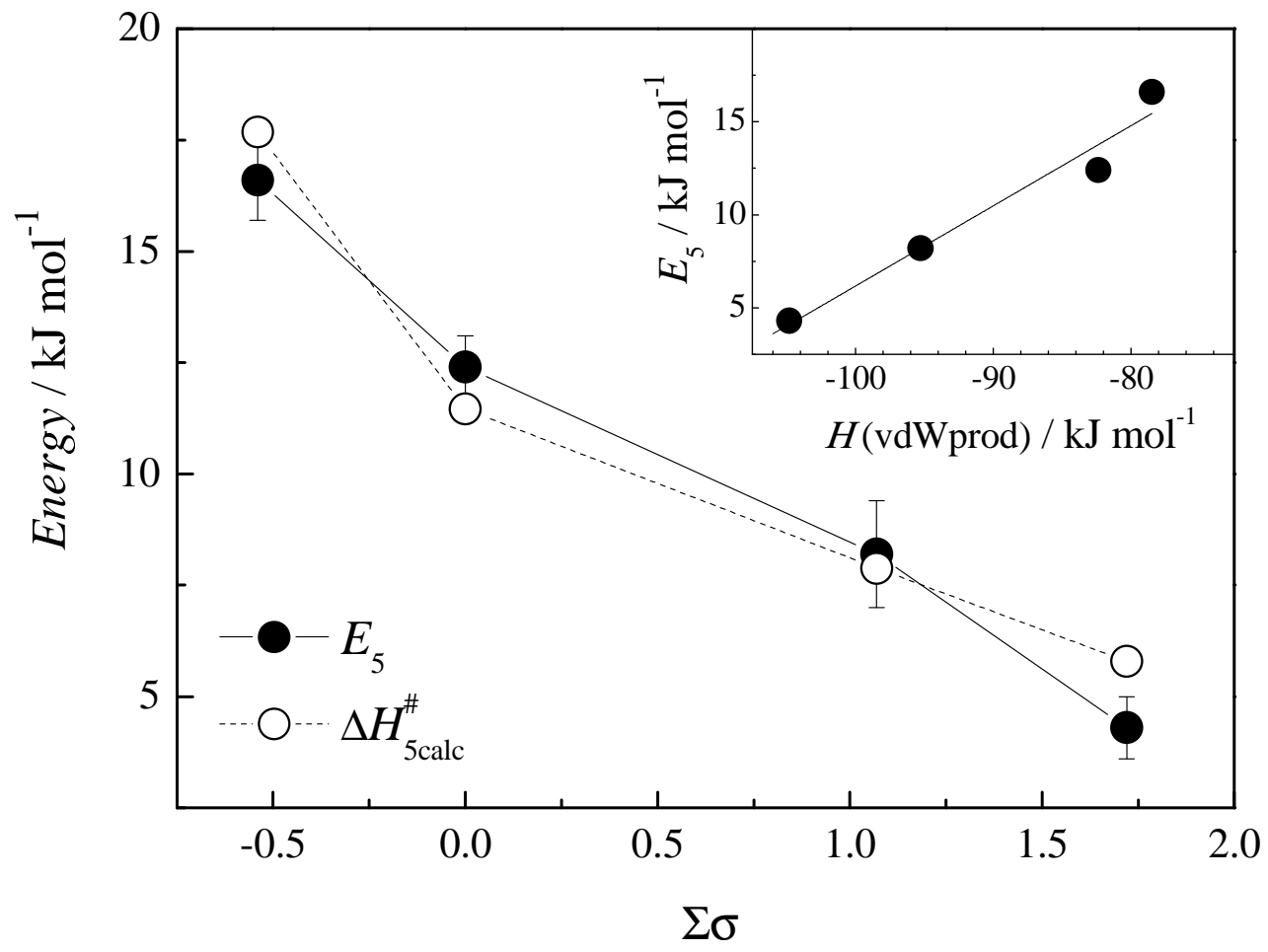

Figure 10. The measured activation energies (filled circles) and the calculated activation enthalpies (open circles, shifted by $-\Delta H_{8}=21.6 \mathrm{~kJ} \mathrm{~mol}^{-1}$ ) of reaction (5) as a function of the overall Hammett parameter of substituents of the four studied benzophenone derivatives.

Inset: The measured activation energy $E_{5}$ as a function of the corresponding reaction enthalpy (formation enthalpy of the product vdW complex). 
Supporting Information. Synthesis methods, details of spectrum, rate coefficient measurements and of electronic structure calculations. This material is available free of charge via the Internet at http://pubs.acs.org.

\section{Corresponding Authors}

*E-nail: demeter.attila@ttk.mta.hu, soos.tibor@ttk.mta.hu, lendvay.gyorgy@ttk.mta.hu

\section{ACKNOWLEDGMENT}

The work was supported by the Hungarian Science Foundation (OTKA T45890 and T77938) and by the National Development Agency (Grant No. KTIA_AIK_12-1-2012-0014). The contribution of the late professor Tibor Bérces, who left us during this project, is greatly acknowledged.

\section{REFERENCES}

(1) Ciamician, G.; Silber, P. Chem. Ber. 1900, 33, 2911.

(2) Bäckstrom, H. L. J.; Sandros K. Acta Chem. Scand. 1960, 14, 48.

(3) Beckett, A.; Porter, G. Trans. Faraday Soc. 1963, 59, 2038.

(4) Pitts, Jr. J. N.; Letsinger, R. L.; Taylor, R. P.; Patterson, J. M.; Recktenwald, G.; Martin R. B. J. Amer. Chem. Soc. 1959, 80, 1068.

(5) Org. Synth. Col. Vol. I p. 90; John Wiley \& Sons Inc., London 1948; Fieser, L. F.; Fieser, M. Organic Chemistry 3th ed. 700; Reinhold Pub. Corp., New. York, 1963.

(6) Pischel, U.; Nau, W. M. Photochem. Photobiol. Sci. 2002, 1, 141.

(7) Zimmerman H. E. Adv. Photochem. 1963, 1, 183; Padwa, A. Tetrahedron Lett. 1964, 3465 . 
(8) (a) Scaiano, J. C. J. Photochem. 1973, 2, 81, and references therein; (b) Wagner, P. J.; Truman, R. J.; Scaiano, J. C. J. Amer. Chem. Soc. 1985, 107, 7093.

(9) Formosinho, S. J.; Arnaut, L. G. Adv. Photochem. 1991, 16, 67; Arnaut, L. G.; Formosinho, S. J.; Da Silva A. M. J. Photochem. 1984, 27, 185.

(10) Görner, H. Photochem. Photobiol. 2006, 82, 801; (d) Görner, H. J. Photochem. Photobiol. A: 2007, 187, 105.

(11) Favaro, G. Chem. Phys. Lett. 1973, 21, 401; Favaro, G. J Photochem. 1981, 15, 89; Favaro, G.; Ortica, F.; Romani, A. J. Photochem. Photobiol. C 2013, 16, 22.

(12) Turro, N. J.; Ramamurthy, V.; Scaiano, J. C. Modern Molecular Photochemistry of Organic Molecules; University Science Books: Sausalito, California, 2010.

(13) Demeter, A.; Bérces, T. J. Phys. Chem. 1991, 95, 1228, and references therein.

(14) Naguib, Y. M. A.; Steel, C.: Cohen, S. G. J. Phys. Chem. 1988, 92, 6574.

(15) Dalicsek, Z.; Pollreisz, F.; Soós, T. Chem. Commun. 2009, 4587.

(16) (a) Demeter, A.; László, B.; Bérces, T. Ber. Bunsenges. Phys. Chem. 1988, 92, 1478; (b) Demeter, A.; Bérces, T. J. Photochem. Photobiol. A 1989, 46, 27, and references therein.

(17) Shah, K. B.; Neckers, D. C. J. Photochem. Photobiol. A 2005, 170, 195; Aloïse, S.; Ruckebusch, C.; Blanchet, L.; Réhault, J.; Buntinx, G.; Huvenne, J. P. J. Phys. Chem. A 2008, 112, 224.

(18) http://physics.nist.gov/PhysRefData/Handbook/Tables/mercurytable2.htm 
(19) Demas, J. N.; Crossby, G. A. J. Phys. Chem. 1971, 75, 991.

(20) Demeter, A.; Ravasz, L.; Bérces, T. J. Phys. Chem. A 2004, 108, 4357; Demeter, A.; Bérces, T. J. Phys. Chem. A 2005, 109, 2043.

(21) (a) Handbook of Photochemistry 3. p. 102; Ed.: Montalti, M.; Credi, A.; Prodi, L.; Gandolfi, M.T.; Taylor \& Francais, Boca Raton, 2006; (b) Merkel, P. B.; Dinnocenzo, J. P. J. Photochem. Photobiol. A 2008, 193, 110.

(22) Becke, A.D. J. Chem. Phys. 1993 98, 1372; Becke, A. D. J. Chem. Phys. 1993, 98, 5648; Lee, C.; Yang, W.; Parr, R. G. Phys. Rev. B 1988, 37, 785.

(23) Zhao, Y.; Lynch, B. J.; Truhlar, D. G. J. Phys. Chem. A 2004, 108, 2715.

(24) Runge, E.; Gross, E. K. U. Phys. Rev. Lett. 1984, 52, 997; Petersilka, M.; Gossmann, U. J.; Gross, E. K. U. Phys. Rev. Lett. 1996, 76, 1212; Casida, M. E. in Recent Advances in Density Functional Methods, Part I; Ed.: Chong D. P. World Scientific, Singapore, 1995; Bauernschmitt, R.; Ahlrichs, R. Chem. Phys. Lett. 1996. 256, 454.

(25) Hochstrasser, R. M.; Noe, L. J. J. Molec. Spectr. 1971, 38, 175; Shimamori, H.; Uegaito, H.; Houdo, K. J. Phys. Chem. 1991, 95, 7664; Correa, A. M. B.; Goncalves, A. S.; de Souza, A. M. T.; Freitas, C. A.; Cabral, L. M.; Albuquerque, M. G.; Castro, H. C.; dos Santos, E. P.; Rodrigues, C. R. J. Phys. Chem. A 2012, 116, 10927.

(26) Demeter, A.; Mile, V.; Bérces, T. J. Phys. Chem. A 2007, 111, 8942.

(27) Lewis, J. E.; Maroncelli, M. Chem. Phys. Lett. 1998, 282, 197.

(28) Winnik, M. A.; Lemire, A. Chem. Phys. Lett. 1977, 46, 283. 
(29) Lehni, M.; Fischer H. Int. J. Chem. Kinet. 1983, 15, 733.

(30) Horváth, I. T.; Mehdi, H.; Fábos, V.; Boda, L.; Mika L. T. Green Chem. 2008, 10, 238.

(31) Kearns, D. R.; Case, W. A. J. Am. Chem. Soc. 1966, 88, 5087.

(32) Wintgens, V.; Valat, P.; Kossanyi, J.; Demeter, A.; Biczók, L.; Bérces, T. J. Photochem. Photobiol. A 1996, 93, 109.

(33) Huang, W.; Zhang, X.; Ma, L. H.; Wang, C. J.; Jiang, Y. B. Chem. Phys. Letts. 2002, 352, 401; Zhang, X.; Sun, X. Y.; Wang, C. J.; Jiang, Y. B. J. Phys. Chem. A 2002, 106, 5577; Zhang, X.; Wang, C. J.; Liu, L. H.; Jiang, Y. B. J. Phys. Chem. B 2002, 106, 12432.

(34) (a) Leight, W. J.; Arnold D. R. J. C. S. Chem. Comm. 1980, 406; (b) Leight, W. J.; Arnold, D. R.; Humphreys, R. W. R.; Wong, P. C. Can. J. Chem. 1980, 58, 2537.

(35) (a) Jaffé, H. H. Chem. Rev. 1953, 53, 191; (b) Brown, H. C.; Okamoto, Y. J. Am. Chem. Soc. 1958, 80, 4979.

(36) Arthur, N. L.; Christie, J. R. Int. J. Chem. Kinet. 1987, 19, 261. 
Table of Contents (TOC) Image

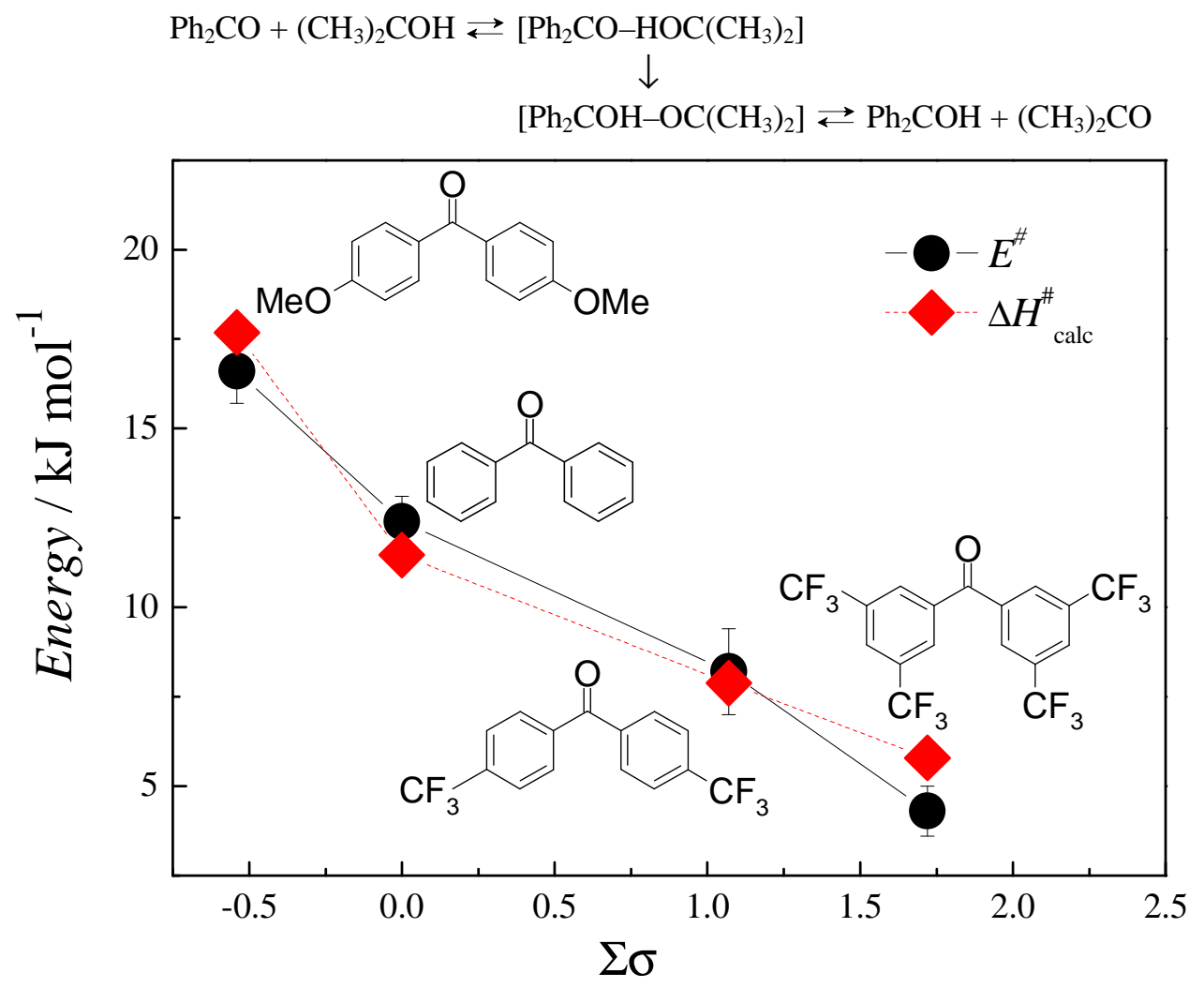

